\title{
Ion energies and currents of Type I and mitigated ELMs in the ASDEX Upgrade far scrape-off layer
}

\author{
M. Kočan ${ }^{1}$, S. Y. Allan², S. Carpentier-Chouchana ${ }^{3}$, P. de Marné ${ }^{1}$, S. Elmore ${ }^{2}$, \\ T. Franke ${ }^{1}$, J. P. Gunn ${ }^{4}$, A. Herrmann ${ }^{1}$, A. Kirk ${ }^{2}$, M. Kubič ${ }^{4}$, T. Lunt ${ }^{1}$, H. W. Müller ${ }^{1}$, \\ R. A. Pitts ${ }^{3}$, V. Rohde ${ }^{1}$ and ASDEX Upgrade Team \\ ${ }^{1}$ Max-Planck-Institut für Plasmaphysik, EURATOM Association, Boltzmannstr. 2, D-85748 Garching, Germany \\ ${ }^{2}$ Culham Centre for Fusion Energy, Abingdon, OX14 3DB, United Kingdom \\ ${ }^{3}$ ITER Organization, Route de Vinon, CS 90 046, 13067 Saint Paul Lez Durance Cedex, France \\ ${ }^{4}$ CEA, IRFM, F-13108 Saint-Paul-lez-Durance, France
}

E-mail: martin.kocan@ipp.mpg.de

\begin{abstract}
New measurements of ion energies and currents in Type I and mitigated ELMs have been carried out in the ASDEX Upgrade far scrape-off layer using a retarding field analyzer (RFA). The ion temperature averaged over an ELM, $T_{i E L M}$ measured 35-60 mm outside the separatrix (i.e. $15-25 \mathrm{~mm}$ in front of the outboard limiter) is in the range of 20 $200 \mathrm{eV}$, which is $5-50 \%$ of the ion temperature at the pedestal top. $T_{i E L M}$ decreases with the separatrix distance with the e-folding length of $\sim 10 \mathrm{~mm}$ measured in the far SOL for a particular set of conditions, and increases with the ELM energy $W_{E L M}$. Lowest $T_{i E L M}$ is measured during mitigated Type I ELMs. Likewise, the ELM-averaged ion current e-folding length $\lambda_{\text {jsat }} \approx 10-20 \mathrm{~mm}$ increases with $W_{E L M}$, similar to the e-folding length of the heat flux density at the RFA probe head during an ELM, monitored by a fast IR camera. The most plausible explanation of observed trends is that on average the filaments of larger ELMs travel faster radially and have less time to dilute by parallel losses along field lines before reaching the far SOL. These observations provide further evidence that the fraction of the ELM energy deposited on the main chamber plasma facing components increases with $W_{E L M}$.
\end{abstract}

PACS: 52.25.Fi, 52.25.Xz, 52.40.Hf, 52.55.Fa, 52.65.-y, 52.70.Ds 


\section{Introduction}

Type I Edge Localized Modes (ELMs) can carry a significant fraction of their energy, $W_{E L M}$ to main chamber plasma facing components (PFC) in divertor tokamaks and can be the dominant source of the first wall thermal load and impurity sputtering (e.g. [1-10]). In ITER, unmitigated Type I ELM energy losses will be an order of magnitude larger compared with present tokamaks. The ELM-wall interaction will be an issue for the first wall lifetime and tritium retention $[11,12]$ (in case of Be PFC) and impurity production $[13,14]$ (if Be will be replaced by tungsten PFCs).

Recognized as a matter of concern for ITER, the ELM-wall interaction has been intensively studied by a number of diagnostics (see references above). These measurements agree that each ELM consists of a number of field aligned filaments, expelled at different toroidal locations on the plasma low field side into the scrape-off layer (SOL). Qualitatively similar ELM features have been reproduced by non-linear MHD simulations [15-17]. As they propagate across the SOL, propelled by electrostatic interchange motions [18], some filaments break into smaller fragments before hitting the first wall as observed in [5]. There, the deposited fraction of the ELM energy increases with $W_{E L M}$ in exchange for smaller relative divertor loading $[2,5,19-21]$. These observations are consistent with the predicted filament radial propagation speed $v_{r} \propto W_{E L M}^{\alpha}$ with $\alpha=0.5$ [18]. The measured $v_{r}$ follows similar trends in some experiments (e.g. [20]), but there are cases in which it does not [22-24], suggesting that the propagation mechanism is not fully understood.

In the SOL, data gathered by Langmuir probes (LPs) reveal the rich temporal structure of ion current and electron temperature throughout an ELM (e.g. [4, 10, 23, 25-31] and references therein). Ion current observed by LPs during ELMs is found to be significantly higher compared to inter-ELM periods, with a somewhat lower increase of the electron temperature [29]. The most obvious explanation is that fast transport parallel to field lines cools the ELM electrons as filaments propagate into the far SOL (defined grossly as a region 2-3 heat flux density e-folding lengths outside the separatrix). The intensity of the ELM-wall interaction is thus determined mainly by the energies carried into the far SOL by less mobile ions in the ELM filaments. 
One of the major drawbacks of a LP is that it does not provide information on the ion energy. Sparse but clear evidence that ELM ions carry a significant fraction of the pedestal energy into the far SOL has been obtained by infrared (IR) thermography combined with LP data [30], and by retarding field analyzers (RFAs) [4, 10, 32]. So far, such measurements were available only for an extremely limited range of plasma conditions. Nonetheless, a similar range of far SOL ELM ion energies has been predicted by simple models of the parallel ELM filament transport $[4,7,10,33]$.

New measurements of ion energy and current in ELMs have been obtained in the ASDEX Upgrade (AUG) far SOL using a RFA and are reported in this paper. Type I ELMs and, for the first time, ELMs mitigated by magnetic perturbations are studied for a range of separatrix distances and $W_{E L M}$. RFA measurements are compared with visible and IR camera observations and interpreted by means of a fluid parallel transport model [33] in a manner similar to that presented in $[4,22,33]$. These measurements help to constrain the models of the ELM transport in the SOL [7, 33-35] and ELM-induced impurity release from the nondivertor PFCs [36], making predictions towards ITER less uncertain.

Plasma pulses in which the RFA measurements were obtained are described in section 2. Following a brief description of the RFA technique in section 3.1, typical time traces acquired by the RFA during ELMs are illustrated and qualitatively compared to fast visiblelight camera observations in section 3.2. In section 4, the RFA-inferred ELM-averaged ion current density and temperature in the far SOL are studied as a function of the separatrix distance and $W_{E L M}$. In the same section, $v_{r}$ averaged over an ELM is estimated from these measurements. RFA data are compared with the thermographic observations of the ELM heat load to the probe head in section 5 and interpreted by a simple fluid model of the parallel ELM filament transport in section 6. Section 7 provides summary and conclusions.

\section{Discharge conditions}

AUG is a divertor tokamak operated with full tungsten coated PFCs [37]. New invessel saddle coils for ELM mitigation (four coils above and four coils below the outer 
midplane) were operational in the present experiment $[38,39]$. RFA measurements were obtained in lower single null H-mode discharges in deuterium with the edge plasma parameters compiled in Table 1. A representative magnetic equilibrium is shown in Fig. 1. Type I ELMs were measured at a neutral beam heating power $P_{N B I}=2.5 \mathrm{MW}$ (with additional electron cyclotron resonant heating of $P_{E C R H}=0.8 \mathrm{MW}$ in discharge series \#5). The ELM size in the plasma pulses \#26160-4 was varied by changing the gas fuelling rate during the flat top phase of the discharge. The ELM mitigation was obtained in discharges with $P_{N B I}=5 \mathrm{MW}$ and $n=2$ odd parity resonant magnetic perturbations at a coil current of $900 \mathrm{~A}$. The small ELMs observed in discharge series 6 will be referred to as 'mitigated' ELMs throughout this paper. In all discharges the ion $\mathbf{B} \times \nabla \mathrm{B}$ drift points downwards, plasma current $I_{p}=1 \mathrm{MA}$, toroidal field $B_{t}=-2.5 \mathrm{~T}$, edge safety factor at $95 \%$ flux surface $q_{95}=4.6-4.8$, major radius $R=1.66-1.69 \mathrm{~m}$, and plasma volume $V_{\text {plasma }}=14 \mathrm{~m}^{3}$. The plasma stored energy prior to the ELM was $W_{\text {plasma }}=280-490 \mathrm{~kJ}$. Throughout this paper, the ELM energy $W_{E L M}$ corresponds to the drop of $W_{\text {plasma }}$ during an ELM. The parallel collisionality at the pedestal top $v_{e}^{*} \approx q_{95} \pi R / \lambda_{e e} \approx 1.5-3$ where $\lambda_{e e} \cong 10^{16} T_{e p e d}^{2} / n_{\text {eped }}(\mathrm{m}$, $\left.\mathrm{eV}, \mathrm{m}^{-3}\right)$ is the electron-electron collisional mean free path evaluated at the pedestal top. All discharges have similar magnetic equilibria which will be discussed later in relation to the parallel magnetic connection lengths in the SOL.

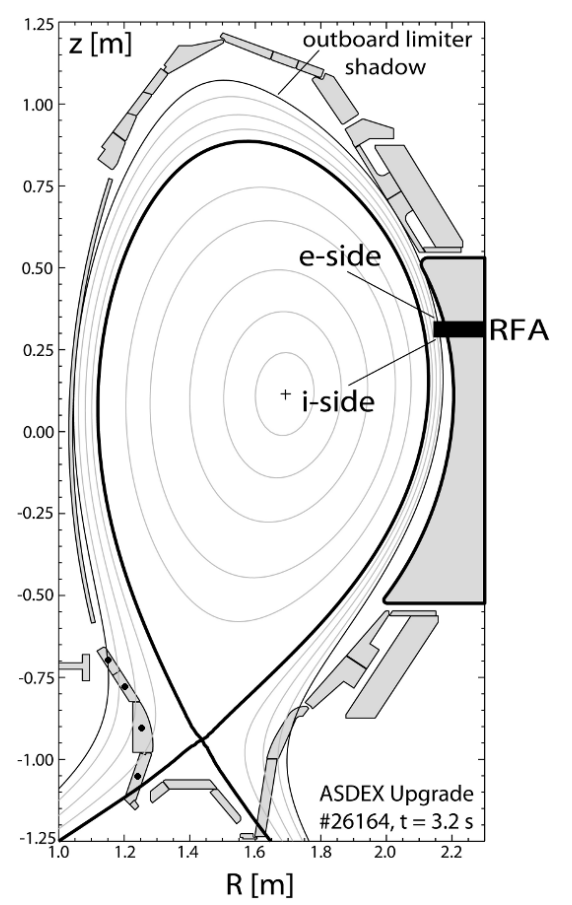

Figure 1. Poloidal cross section of AUG. $i$-side / e-side indicates the side of a bidirectional RFA. The RFA axis is aligned with the local magnetic field. The i-side (eside) RFA faces the outer (inner) divertor along the magnetic field lines. Dots indicate a complete array of inner divertor tiles used for the measurements of the inner divertor shunt current $I_{d i v}$. 


\begin{tabular}{llllllllll}
\hline Series & Discharge & $\begin{array}{l}T_{\text {iped }} \\
{[\mathrm{eV}]}\end{array}$ & $\begin{array}{l}T_{\text {eped }} \\
{[\mathrm{eV}]}\end{array}$ & $\begin{array}{l}n_{\text {eped }} \\
{\left[10^{19} \mathrm{~m}^{-3}\right]}\end{array}$ & $\begin{array}{l}W_{\text {ELM }} \\
{[\mathrm{kJ}]}\end{array}$ & $\begin{array}{l}W_{\text {ELM }} / W_{\text {plasma }} \\
{[\%]}\end{array}$ & $\begin{array}{l}W_{\text {ELM }} / W_{\text {ped }} \\
{[\%]}\end{array}$ & $\begin{array}{l}f_{\text {ELM }} \\
{[\mathrm{Hz}]}\end{array}$ & $\begin{array}{l}\Delta r_{\text {lim }} \\
{[\mathrm{mm}]}\end{array}$ \\
\hline 1 & $26208 \& 9$ & 350 & 250 & $7.6-7.8$ & $27 \pm 6$ & $7 \pm 2$ & 19 & $40 \pm 8$ & $20-25$ \\
2 & 26164 & 300 & 250 & $6.9-7.1$ & $28 \pm 10$ & $10 \pm 4$ & 22 & $45 \pm 16$ & 15 \\
3 & $26160 \& 2$ & 400 & 350 & $6.8-7.0$ & $34 \pm 12$ & $10 \pm 3$ & 22 & $46 \pm 19$ & 15 \\
4 & $26160 \& 2 \& 4$ & 350 & 300 & 7.3 & $36 \pm 7$ & $11 \pm 2$ & 23 & $46 \pm 7$ & 15 \\
5 & 26164 & 400 & 400 & $6.8-7.1$ & $56 \pm 7$ & $15 \pm 2$ & 31 & $49 \pm 14$ & 15 \\
$6 *$ & $26208 \& 9$ & 450 & 350 & $7.6-7.8$ & $* *$ & $* *$ & $* *$ & $245 \pm 123$ & $20-25$ \\
\hline$*$ & & & & & & &
\end{tabular}

Table 1. From left to right: index of the discharge series preserved throughout the paper, discharge number, ion and electron temperatures (CXRS, ECE and Thomson scattering diagnostics) and plasma density (interferometer, lithium beam, Thomson scattering) at the pedestal top ( $\sim 1.5 \mathrm{~cm}$ inside the separatrix), plasma energy lost per ELM (absolute, relative to $W_{\text {plasma, }}$ and the pedestal energy normalized to the plasma volume $W_{\text {ped }}=\frac{3}{2} n_{\text {eped }}\left(T_{i p e d}+T_{\text {eped }}\right) V_{\text {plasma }}$ using the usual convention [40]) and the approximate ELM frequency $f_{E L M}$. Also indicated is the radial distance in front of the outboard limiter, $\Delta r_{\text {lim }}$ at which the RFA sensor was maintained during the reciprocation.

\section{RFA set up and typical ELM signals}

\subsection{RFA set up}

A bidirectional RFA used in this experiment is shown in Fig. 2. The probe is mounted on the horizontal scanning drive located on the plasma outboard side, $31 \mathrm{~cm}$ above the machine equatorial plane as indicated in Fig. 1. The RFA axis is aligned with the local magnetic field, meaning that the probe is sensitive to the ion velocity component parallel to $\mathbf{B}$. The "i-side" RFA faces in the direction towards the outer divertor and the "e-side" RFA is looking along field lines into the inner divertor, referring to the ion and electron $\mathbf{B} \times \nabla B$ drift directions. As shown in Fig. 3, each of the two analysers consists of a set of semi-permeable grids and a collector, separated from the plasma by a thin plate in which a narrow slit is cut. The slit plate serves to protect the delicate grids from intense plasma irradiation, while admitting enough ions to produce a measurable current on the collector $\left(I_{c}\right)$. The slit plate is 
perpendicular to the magnetic field direction so that the RFA is sensitive to the parallel velocity of incident ions. The slit plate is biased negatively $\left(V_{s p}\right)$ to repel most incident electrons back into the plasma and to measure the ion current density $I_{s a t}$. A fraction of the incident ions are transmitted through the slit into the analyzer where they encounter the first grid, labelled as "grid 1", to which a positive voltage $V_{g 1}$ is applied. The ions that have enough kinetic energy to overcome $V_{g 1}$ then proceed to a second grid, labelled as "grid 2", biased to high negative voltage $\left(V_{g 2}\right)$, and placed between grid 1 and the collector. Grid 2 is used to repel any remaining incident electrons that are energetic enough to penetrate $V_{s p}$, as well as to suppress secondary electrons emitted inside the analyzer.

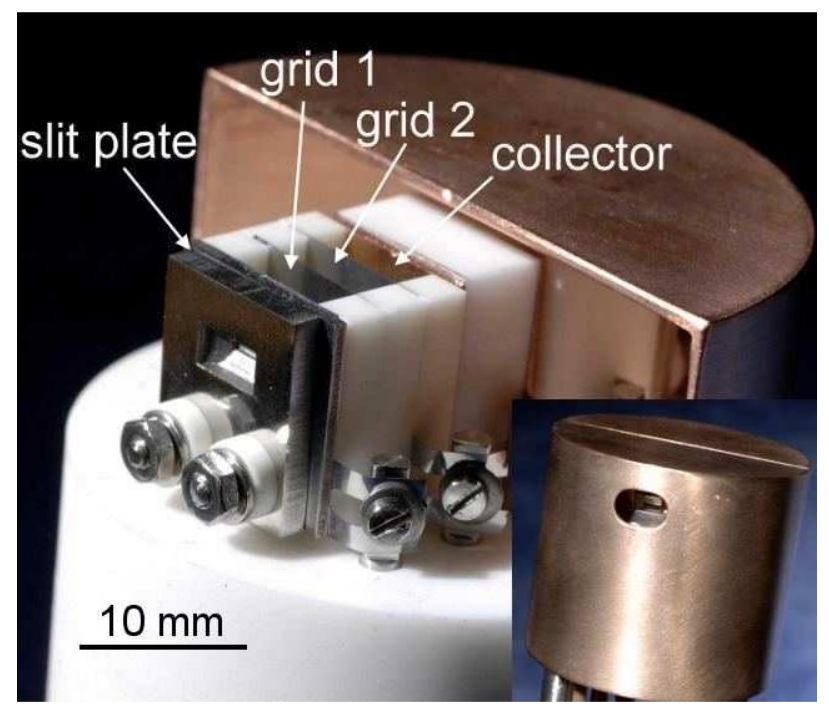

Figure 2. Photograph of the RFA probe head covered by a grounded copper cage. The cage (inset panel) is used to reduce the pick-up noise during ELMs. Half of the cage is removed, showing one of the two identical analyzers mounted in a Mach probe arrangement. The entire probe head is protected by a graphite housing.

Bias voltages applied to RFA electrodes are compiled in Table 2. The same voltages were applied to both analyzers. The voltage $V_{s p}$ is set to repel most ELM electrons (in the AUG far SOL the ELM-averaged electron temperature $T_{e E L M} \approx 15 \mathrm{eV}$ [31]). All RFA signals are measured at an acquisition frequency of $2 \mathrm{MHz}$. The ion current density $j_{s a t}=I_{s a t} / A_{s p}$, where $A_{s p}=16 \mathrm{~mm}^{2}$ is the slit plate collecting area. The entire probe head is protected by a graphite housing with the outer diameter $d=6.2 \mathrm{~cm}$. The leading edge of the housing is about $15 \mathrm{~mm}$ closer to separatrix compared with the RFA sensors. 


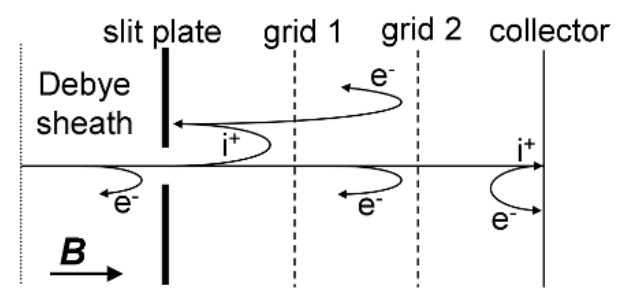

Figure 3. Schematic of the RFA and the bias voltages applied to the electrodes. All voltages refer to torus ground.

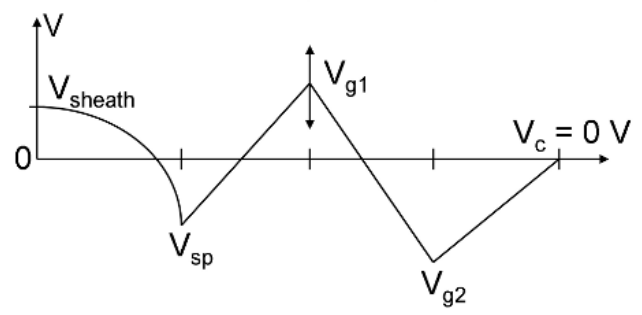

\begin{tabular}{lllll}
\hline Discharge & $V_{s p}[\mathrm{~V}]$ & $V_{g 1}[\mathrm{~V}]$ & $V_{g^{2}}[\mathrm{~V}]$ & $f_{g I}[\mathrm{~Hz}]$ \\
\hline 26160 & -90 & $50-300$ & -150 & 10 \\
26162 & -100 & $80-400$ & -160 & 5 \\
26164 & -100 & $80-400$ & -160 & 4 \\
26208 & -150 & $70-230$ & -190 & 7 \\
26209 & -150 & $60-180$ & -190 & 10 \\
\hline
\end{tabular}

Table 2. Bias voltages applied to RFA slit plate, grid 1 and grid 2. All voltages refer to torus ground. $f_{g 1}$ is the sweeping frequency of $V_{g 1}$.

Up to four reciprocations were performed in each shot. For each reciprocation the RFA was maintained at fixed outboard midplane separatrix distance $\Delta r_{\text {sep }}$ (and $\Delta r_{\text {lim }}$ from Table 1) for $t_{\text {target }}=100-200 \mathrm{~ms}$ and about 5-10 Type-I ELMs or 25-50 mitigated ELMs were captured randomly at different $V_{g 1}$. The measurements were obtained for $\Delta r_{\text {sep }} \approx 35-60$ $\mathrm{mm}$. The voltage $V_{g 1}$ was swept at a few $\mathrm{Hz}$ to make at least one full voltage ramp while holding the probe at the target position, but to keep $V_{g 1}$ approximately constant during an ELM which typically lasts a few ms.

\subsection{RFA time traces during ELMs}

Typical RFA signals measured during Type I ELMs (discharge series \#4) at $\Delta r_{\text {sep }}=35 \mathrm{~mm}$ are illustrated in Fig. 4. Also plotted is $W_{\text {plasma }}$ as well as the current to the inner divertor $I_{d i v}$ (standard ELM marker in AUG) measured by the series of shunt resistors embedded in the tile mountings [41]. $I_{d i v}$ is the sum of currents measured by a complete array 
of four inner divertor tiles (Fig. 1) located at two toroidal sectors and multiplied by a number of tiles in a given row, resulting in a total effective collecting (i.e. the inner divertor) area of $3.9 \mathrm{~m}^{2}$. The RFA collector current is effectively zero in the inter-ELM periods meaning that $V_{g 1}$ is large enough to repel background ions. The current to the collector measured during an ELM decreases with increasing $V_{g 1}$. At $V_{g 1}=325 \mathrm{~V}$ the ELM ions are almost completely suppressed from the i-side collector. The e-folding voltage of the i-side collector current is approximately $100 \mathrm{~V}$, meaning that the ELM ions reach $\Delta r_{\text {sep }}=35 \mathrm{~mm}$ with the typical energies of about $100 \mathrm{eV}$. This would correspond to about $30 \%$ of the ion temperature at the pedestal top (see Table 1).
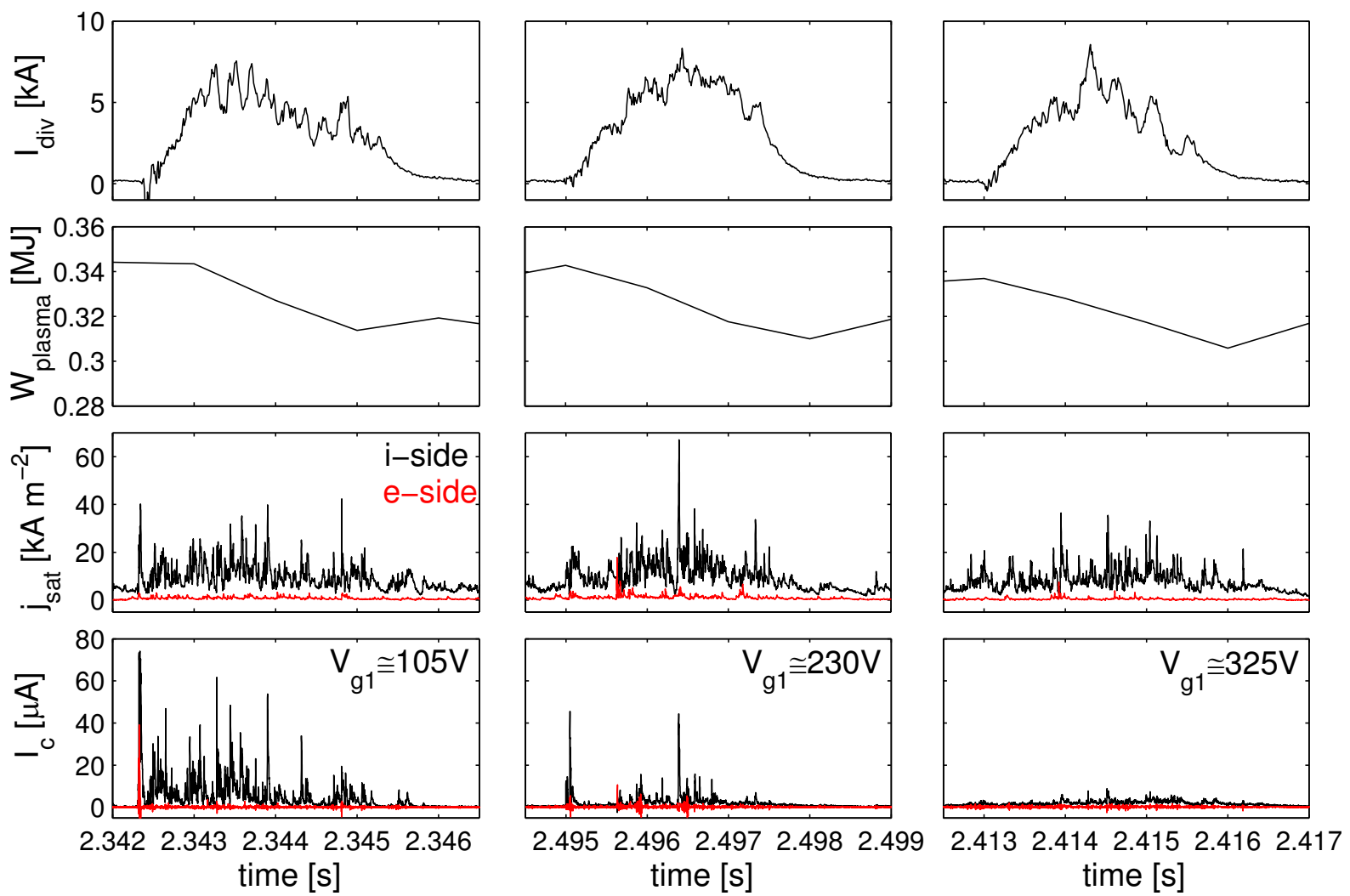

Figure 4. Time evolution of the current to the inner divertor $I_{d i v}, W_{\text {plasma }}$ and the currents measured by the RFA during three similar Type I ELMs. Measurements were obtained at $\Delta r_{\text {sep }}=35 \mathrm{~mm}$ (\#26162, discharge series \#4). i-side / e-side indicates the side of the RFA (Fig. 1). Also indicated is $V_{g 1}$ applied at the ELM.

The time evolution of $j_{s a t}$ and $I_{c}$ measured during an ELM have a rich dynamic structure (partially smeared out in the $I_{d i v}$ signal because of the large collecting surface 
involved) which is well known from earlier probe measurements $([4,10,23,25-31]$ and references therein). Such filamentary structure has previously been observed on the AUG divertor target plates using the thermographic diagnostics [42, 43]. Each ELM time trace seen in Fig. 4 consists of a number of bursts lasting several tens of $\mu$, separated by up to several hundred $\mu$ s, interwoven with smaller spikes. In most ELMs, the large current bursts are not clearly separated, indicating that any filament statistics (e.g. number of filaments per ELM, filament duration at half maximum and filament separation) would strongly depend on the filament detection thresholds. Fig. 4 also illustrates a general observation that even in similar ELMs, the time evolution of $j_{\text {sat }}$ does not follow a universal pattern throughout an ELM. In some ELMs the amplitude of the current bursts decreases during the ELM, whilst in many events the amplitude changes randomly throughout, as already pointed out for example in [30]. The first observation would indicate that more intense filaments travel faster radially and are less depleted by parallel losses before reaching the probe as suggested e.g. in [24]. The latter observation would reflect the stochastic nature of an ELM: each filament may be ejected into the SOL at slightly different radial position, some filaments may disintegrate before reaching the far SOL $[5,18]$ (which in turn may affect their time of flight to the probe) or overlap when striking the RFA, producing a time trace unique to each ELM. 

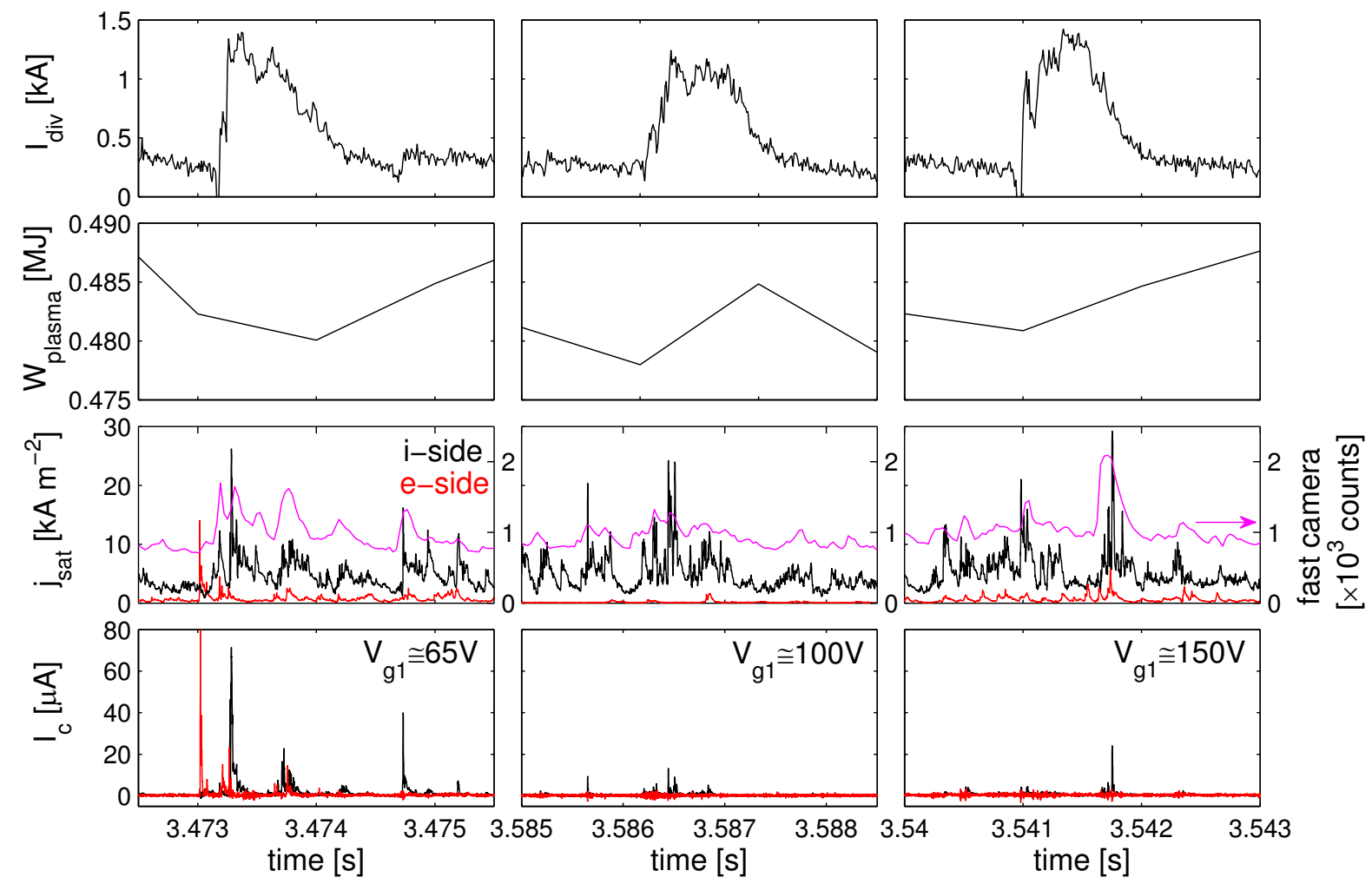

Figure 5. Composite similar to Fig. 4, showing the RFA signals measured at $\Delta r_{\text {sep }}=49 \mathrm{~mm}$ during three similar mitigated ELMs (\#26209, discharge series \#6). The change of $W_{\text {plasma }}$ is below the detection limit. Also plotted is the signal from the fast visible camera, at the ROI centred at the RFA probe (Fig. 6).

In Fig. 5, characteristic features observed by the RFA in mitigated ELMs at $\Delta r_{\text {sep }}=49$ $\mathrm{mm}$ are shown. The bursts of $I_{d i v}$ are almost an order of magnitude smaller, with the duration a factor of $\sim 2$ shorter compared with Type I ELMs. The change of $W_{\text {plasma }}$ due to an ELM is at most a few $\mathrm{kJ}$. The filamentary structure of $j_{\text {sat }}$ in mitigated ELMs is qualitatively similar to Type I ELMs. In contrast to Type I ELMs, however, large bursts of $j_{\text {sat }}$ are observed throughout the ELM cycle (including the inter-ELM periods), though, in common with Type I ELMs, the largest bursts are typically correlated with those of $I_{d i v}$. In mitigated ELMs, almost all ions are repelled from the collector already at $V_{g 1} \cong 100 \mathrm{~V}$. This can be explained by the combination of larger $\Delta r_{\text {sep }}$ in Fig. 5 compared with Fig. 4, and, as will be shown in Sec. 4.2, by lower characteristic ELM ion energies in the far SOL in the case of mitigated ELMs. 
One can notice from figures 4 and 5 that very small currents are measured during ELMs by the e-side RFA. As can be seen from Fig. 1, in the present experiment, the magnetic flux surfaces were not perfectly aligned with the edge of the outboard limiter (OL). As a consequence, the upper edge of the OL casts a shadow on the e-side RFA and shortens the magnetic connection length between the probe and the nearest surface to $L_{/ /} \approx 1-2 \mathrm{~m}$, even though the RFA sensors are radially further in than the limiter edge (table 1). As a result, the ELM particles entering the flux tube between the probe and the OL get quickly depleted by parallel losses $\propto c_{s} / L_{/ /}$with $c_{s}$ the ion sound speed, making the measurements by the e-side RFA effectively useless. In what follows we focus on the measurements by the i-side RFA with "i-side" dropped for brevity.

Measurements from a visible-light camera viewing the RFA were acquired in discharge \#26209 in which the time traces of mitigated ELMs plotted in Fig. 5 were obtained. Images $(256 \times 256$ pixels) were taken with an exposure time of $29 \mu$ s and $33 \mathrm{kHz}$ framing rate. Typical background-subtracted snapshots taken during a Type I ELMy phase of the discharge number \#26209 are illustrated in Fig. 6. Frames 1-3 feature multiple field-aligned filaments of light near the outer wall in front of the viewport and at the inner divertor region. Also shown in Fig. 6 are the time traces of the light intensity averaged over the region of interest (ROI) at the RFA probe and $j_{\text {sat }}$ measured simultaneously by the RFA. The ROI $(5 \times 5$ pixels corresponding to $\sim 2 \times 2 \mathrm{~cm}^{2}$ at the RFA probe) coincides with the center of the bottom part of the RFA housing, which is seen as a bright spot in images 1 and 3 . The light emission at the ROI originates from the deuterium recycling and the ionization of carbon eroded from the probe housing, so that the intensity of the light emission can be linked to that of an ELM filament ion current density. Although the filament fine structure is smeared out on the camera signal due to insufficient time resolution, current bursts are well reproduced by the camera signals. Interestingly, even when the visible light emission in the ROI is strongest, no filaments are observed along the field line intersecting the leading edge of the RFA at $\Delta r_{\text {sep }}=30 \mathrm{~mm}$ which is most likely because of the low neutral density on the flux tubes in that region. 

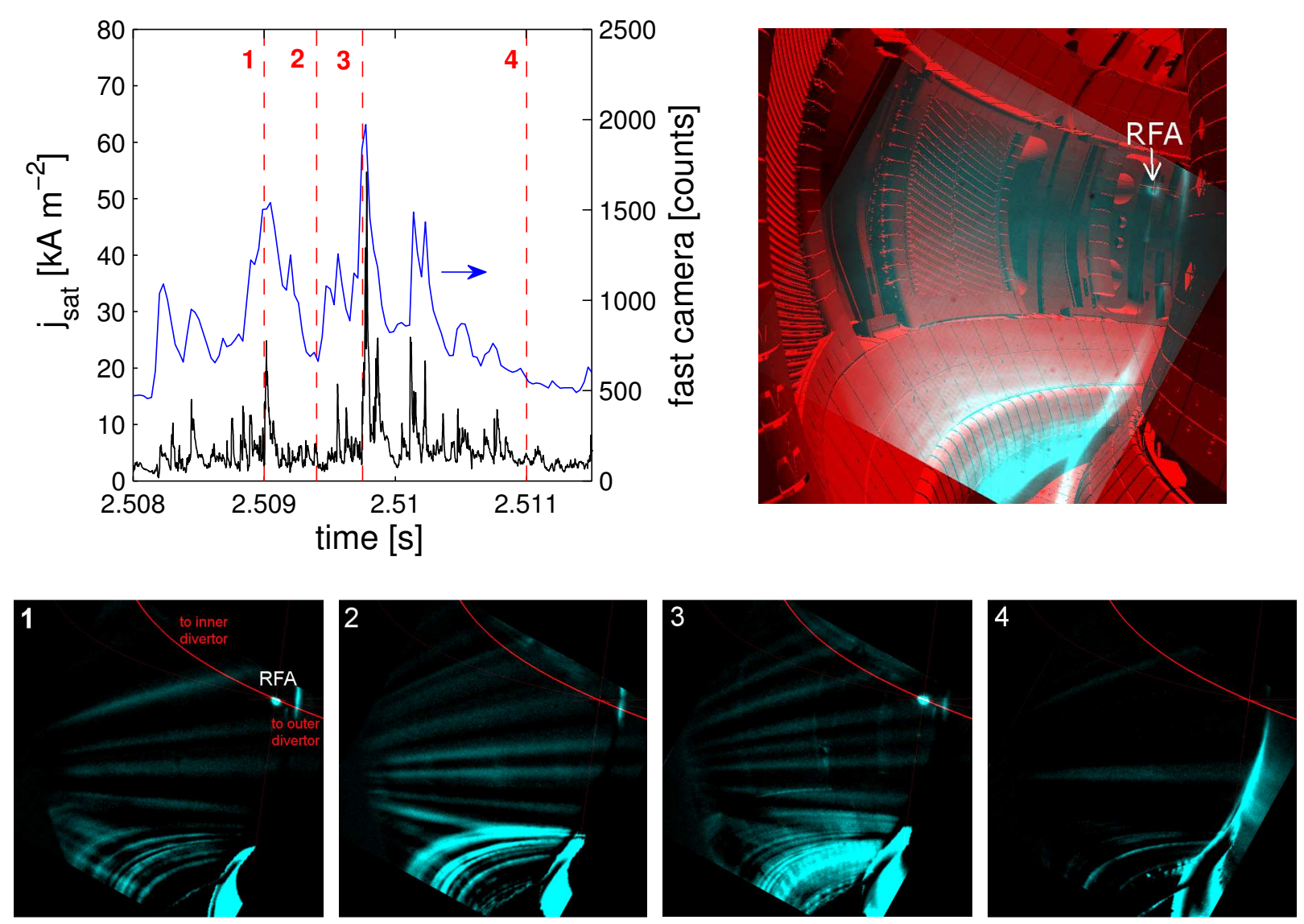

Figure 6. Top left: time evolution of $j_{\text {sat }}$ measured by the RFA at $\Delta r_{\text {sep }}=45 \mathrm{~mm}$ and the visible light intensity at the RFA probe housing monitored by the fast camera during a single Type I ELM in discharge \#26209. The camera view is shown at top right. The leading edge of the RFA housing is at $\Delta r_{\text {sep }}=30 \mathrm{~mm}$ corresponding to $41 \mathrm{~mm}$ in front of the OL. Vertical dashed lines indicate the times when the background-subtracted images shown below were acquired. Also shown is the field line intercepting the RFA.

\section{ELM-averaged ion current density and temperature}

Although the filamentary structure is unique to each ELM, as seen from figures 4 and 5, similar ELMs (i.e. similar $W_{E L M}, W_{\text {plasma }}, I_{d i v}$, etc.) are on average characterized by very similar ion currents. Therefore, the aspects of the radial transport of ions and their typical energies in the ELM filaments can be obtained from the ELM-averaged ion current density $\left\langle j_{s a t}\right\rangle_{E L M}$ and collector currents $\left\langle I_{c}\right\rangle_{E L M}$. This approach is particularly useful for estimating the ELM-averaged ion temperature $T_{i E L M}$ (obtained with the RFA in less transient conditions 
from the variation of $I_{c}$ with $V_{g 1}$ ), since the collector current measured during an ELM changes on a much faster timescale than $V_{g 1}$ can be swept. (An estimate of $T_{i E L M}$ on the filament time scale could be obtained by comparing $I_{c}$ with the fraction of $j_{\text {sat }}$ transmitted through the RFA slit [10]. However, the peculiar dependence of the ion slit transmission factor on the sheath potential and the incident ion energy [44] make such estimates very uncertain.) The particle and energy content can strongly differ in consecutive filaments of a single ELM, raising the question of how meaningful the assignment of average quantities to such a dynamic structure can be. However, given the stochastic nature of ELM wall loading [45], it is the average current and energy carried by ELM ions across the SOL which is of practical interest when addressing the ELM-wall interaction issues.

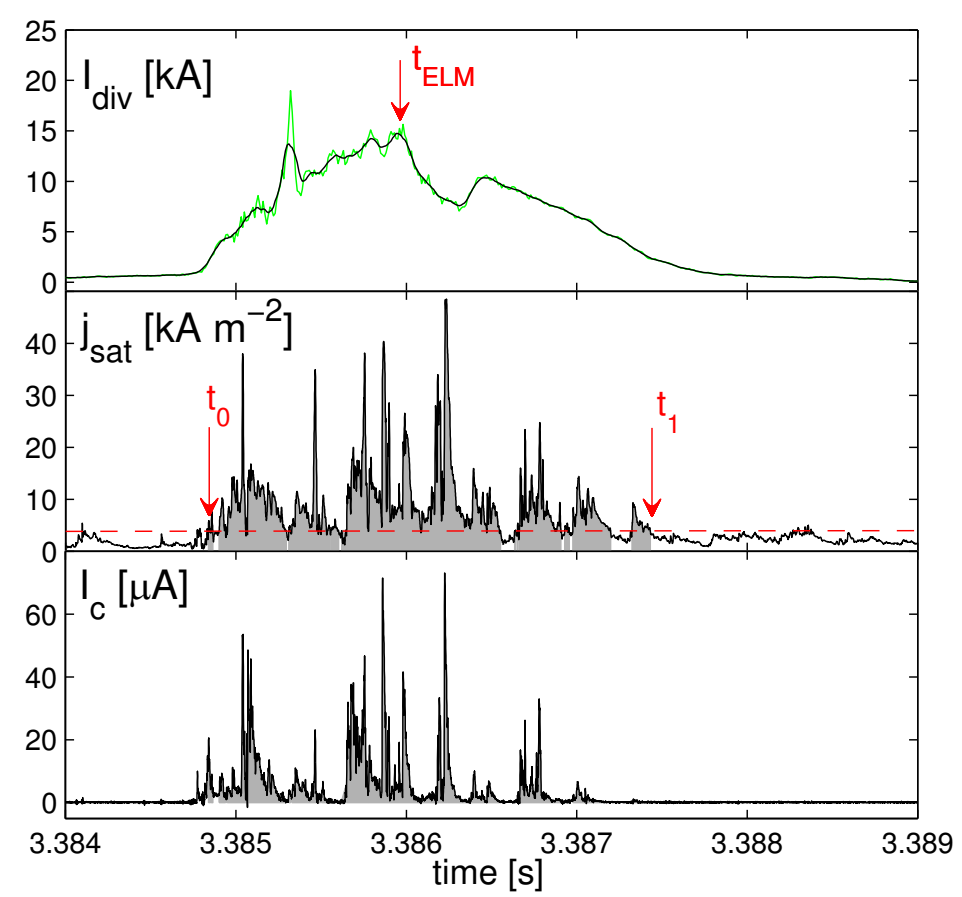

Figure 7. From top to bottom: time evolution of $I_{d i v}, j_{\text {sat }}$ and $I_{c}$ during a single Type $I$ ELM (\#26164, $\left.\Delta r_{\text {sep }}=48 \mathrm{~mm}\right)$ by the i-side analyzer. $j_{\text {sat }}$ is scanned over $-2 m s<t-t_{E L M}<3 \mathrm{~ms}$ (with $t_{E L M}$ at the maximum of the smoothed $I_{\text {div }}$ signal). Shaded areas indicate the portions of $j_{\text {sat }}$ and $I_{c}$ above the ELM ion current detection threshold (dashed) for at least $10 \mu \mathrm{s}$ (see text). The "ELM ion current" is averaged from $t_{0}$ to $t_{1}$. 
The ELM-averaged quantities $\left\langle j_{\text {sat }}\right\rangle_{E L M}$ and $\left\langle I_{c}\right\rangle_{E L M}$ are obtained using the procedure illustrated in Fig. 7. The ELMs are identified from the peaks of $I_{d i v}$, smoothed with the span of $100 \mu$ s to leave out the isolated current bursts due to particular filaments. For each ELM, the time when such a peak is detected is labeled as $t_{E L M} \cdot j_{\text {sat }}$ is scanned over an arbitrarily chosen time window $-2 \mathrm{~ms}<t-t_{E L M}<3 \mathrm{~ms}$ for Type I ELMs and $-1 \mathrm{~ms}<t-t_{E L M}<1.5 \mathrm{~ms}$ for mitigated ELMs. The time when $j_{\text {sat }}$ exceeds the detection threshold $\left\langle j_{\text {sat }}\right\rangle_{\text {back }}+3 \cdot \operatorname{std}\left\langle j_{\text {sat }}\right\rangle_{\text {back }}$ (with $\left\langle j_{\text {sat }}\right\rangle_{\text {back }}$ the average inter-ELM ion current density) for more than $10 \mu \mathrm{s}$, containing at least one data point larger than $j_{\text {satback }}+6 \cdot \operatorname{std}\left(j_{\text {satback }}\right)$ within that sequence, is labeled as $t_{0}$. The end of the last such burst within the time window is labeled as $t_{1} \cdot\left\langle j_{s a t}\right\rangle_{E L M}$ is given by the mean of all data points within $t_{0} \rightarrow t_{1}$ (excluding portions of the signal with $j_{\text {sat }}<\left\langle j_{\text {sat }}\right\rangle_{\text {back }}+3 \cdot s t d\left\langle j_{\text {sat }}\right\rangle_{\text {back }}$ for more than $\left.10 \mu \mathrm{s}\right)$, reduced by $\left\langle j_{\text {sat }}\right\rangle_{\text {back }}$. The mean of the collector signal for the same time points equals to $\left\langle I_{c}\right\rangle_{E L M}$.

\subsection{ELM-averaged ion current density}

Fig. 8 illustrates for selected discharge series the radial profiles of $\left\langle j_{s a t}\right\rangle_{E L M}$. Each data point corresponds to a single ELM. Note that $\left\langle j_{s a t}\right\rangle_{E L M}$ obtained for similar ELMs measured at the same $\Delta r_{\text {sep }}$ is well reproducible. Also plotted are the corresponding profiles of $L_{/ /}$between the RFA and outer divertor obtained from the magnetic field lines tracing. Note that $L_{/ /}$is roughly constant for all discharge series. Despite the variation of the radial efolding length $\lambda_{\text {jsat }}$, Fig. 9, the values of $\left\langle j_{s a t}\right\rangle_{E L M}$ measured for different discharge series are surprisingly similar.

In Fig. $9, \lambda_{\text {jsat }}$ measured in the discharge series \#1-\#6 is plotted against $W_{E L M}$. In this figure (as well as in the following figures) mitigated ELMs are plotted as full symbols to highlight that they are different type of ELM-like events compared with unmitigated Type I ELMs [39]. If the mitigated ELMs are ignored in Fig. 9, there is a tendency for $\lambda_{j s a t}$ to increase with $W_{E L M}$. A simple balance between the characteristic filament parallel sink rate $c_{s} / L_{/ /}$and perpendicular transport time $\lambda_{j s a t} / v_{r}[21,23,24]$, 


$$
v_{r} \approx \lambda_{j s a t} c_{s} / L_{/ /}
$$

can be used to relate $\lambda_{j s a t}$ and $v_{r}$. Fig. 9 thus suggests that on average the radial speed with which the ELM filaments travel across the SOL increases with the ELM size. The same conclusion was reached from experimental observations in DIII-D [46] and JET [21]. We return to this point in the following sections.

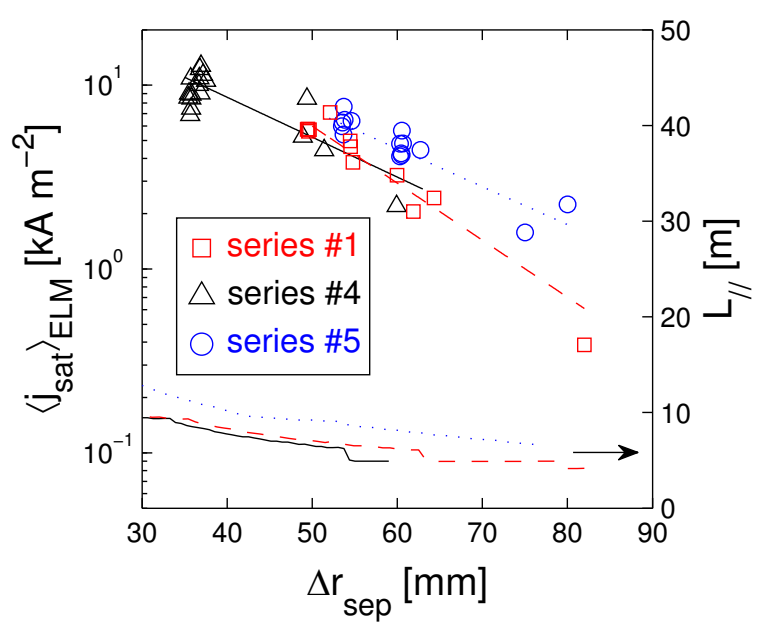

Figure 8. ELM-averaged ion current density measured by RFA in the selected discharge series from Table 1, plotted against $\Delta r_{\text {sep }}$. Guide lines correspond to the ion current density e-folding lengths $\lambda_{\text {jsat. }}$ Also shown are the corresponding profiles of the parallel connection length between the i-side RFA and the outer divertor.

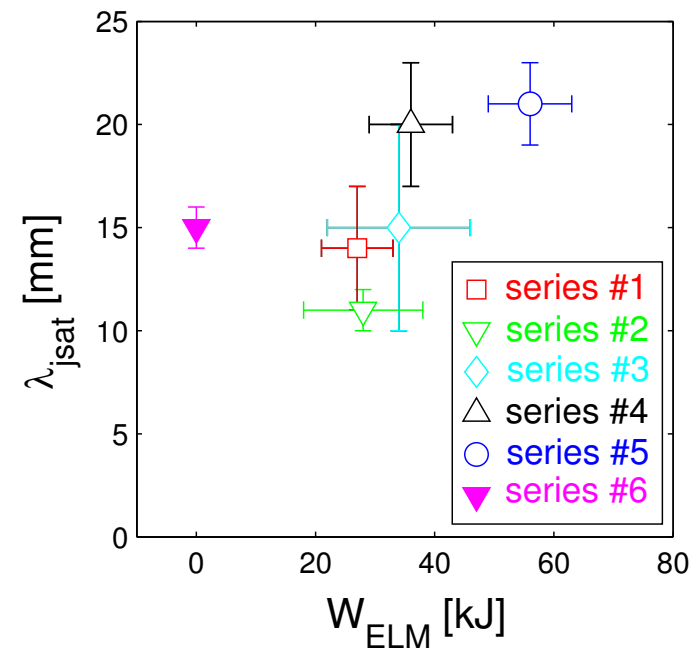

Figure 9. Radial e-folding length of the ELM ion saturation current density $\lambda_{\text {jsat }}$ plotted against the energy lost per ELM. Full symbol: mitigated ELMs.

\subsection{ELM ion temperature}

It is a standard practice when interpreting RFA signals ([47] and references therein) to assume that SOL ions have drifting Maxwellian distribution of parallel speeds and to equate 
the RFA e-folding voltage to ion temperature $T_{i}=-\left(d \ln I_{c} / d V_{g 1}\right)^{-1}$. Given the high $v_{e}^{*}$ in the present discharges stated in Sec. 2, this assumption is used here for ELM ions. Gathering $\left\langle I_{c}\right\rangle_{E L M}$ measured for similar ELMs at constant $\Delta r_{\text {sep }}, T_{i E L M}$ can be obtained from the exponential fit to the filament current-voltage $(I-V)$ characteristic $\left(\left\langle I_{c}\right\rangle_{E L M}\right.$ plotted against $\left.V_{g 1}\right)$. This procedure is illustrated in Fig. 10 where $T_{i E L M}$ is obtained for three different groups of ELMs measured at $\Delta r_{\text {sep }} \cong 48 \mathrm{~mm}$; Type I ELMs characterized by different $W_{E L M}$ and mitigated ELMs. For this particular set of conditions, $T_{i E L M}$ varies from 28 to $155 \mathrm{eV}$.
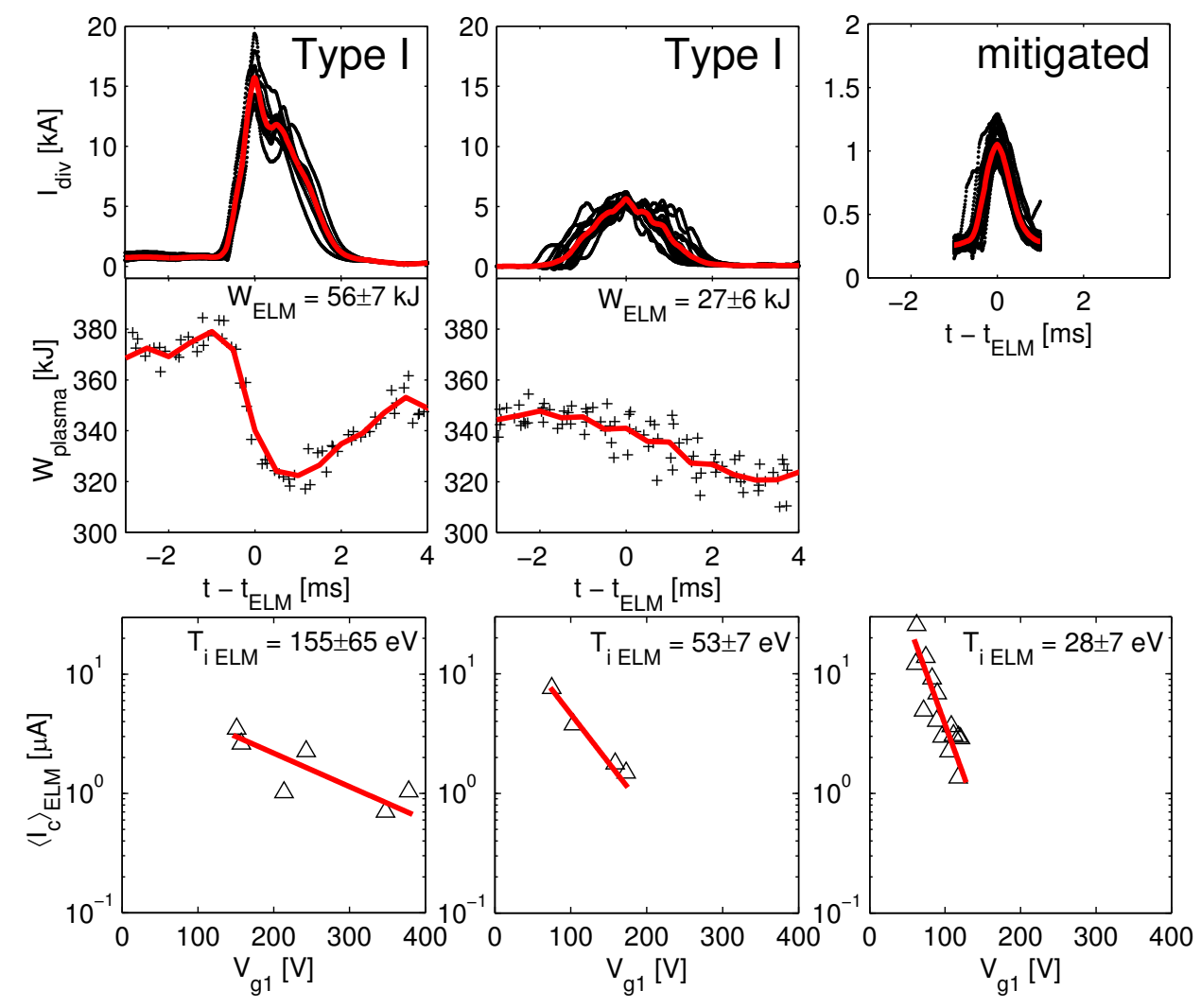

Figure 10. Measurements of $T_{i E L M}$ for three different groups of ELMs at $\Delta r_{\text {sep }} \cong 48 \mathrm{~mm}$. From left to right: Type I ELMs characterized by different $W_{E L M}$ and mitigated ELMs. From top to bottom: coherent average of $I_{\text {div }}, W_{\text {plasma }}$ with the reference time $t_{E L M}$, and the ELM ion I-V characteristics. Each data point on the I-V characteristic corresponds to a single ELM. The exponential fit to the I-V characteristics gives $T_{i E L M}$. The drop of $W_{\text {plasma }}$ is unmeasurable in mitigated ELMs. Note the different scale of $I_{\text {div }}$ for mitigated ELMs. 


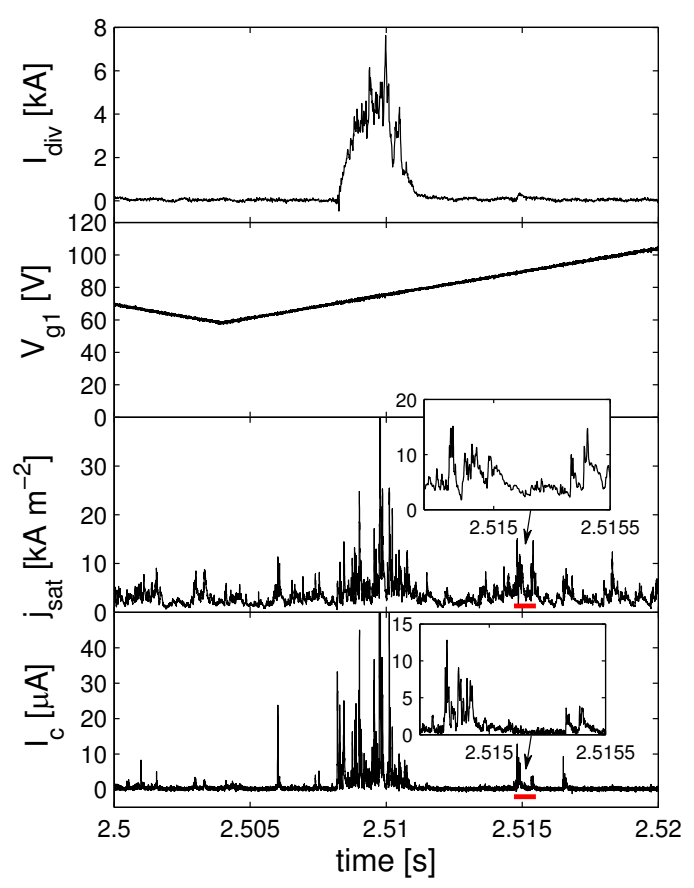

Figure 11. Time evolution of $I_{d i v}$ and the RFA signals measured at $\Delta r_{\text {sep }} \cong 48 \mathrm{~mm}$ in the discharge \#26209 (series \#1). The signals comprise a single ELM at $t \approx 2.51$ s. Inset pannels show details of selected bursts of $j_{\text {sat }}$ and $I_{c}$ observed between ELMs.

It would have been interesting to compare $T_{i E L M}$ with the ion temperature measured at the same radial position between ELMs. Unfortunately, as seen from Fig. 11 (which shows the RFA time traces measured in the discharge number \#26209, series $\# 1, \Delta r_{\text {sep }} \cong 48 \mathrm{~mm}$ ), even the lowest range of $V_{g 1}$ repels most inter-ELM ions from the collector, indicating that the inter-ELM ion temperature in the far SOL is much lower compared with $T_{i E L M}$. This is consistent with the results reported in Ref. [10], which showed initial RFA measurements in AUG in the Type-I ELMy H-mode discharge similar to those in the present experiment. In Ref. [10], $V_{g 1}$ was varied in the range of 0-200 V, which allowed an estimate of the interELM ion temperature $\sim 10 \mathrm{eV}$ at $\Delta r_{\text {sep }} \cong 48 \mathrm{~mm}$. Interestingly, as can be seen from Fig. 11, the collector current measured between ELMs features intermittent positive bursts which are synchronized with the bursts of $j_{\text {sat }}$. These current bursts can be associated with turbulent plasma filaments passing the probe, as observed earlier e.g. in Ref. [48]. The fact that the bursts of $I_{c}$ are observed at $V_{g 1}$ when the collector current is normally absent indicates that 
the turbulent filaments carry ions which are more energetic than those of the surrounding background plasma. Investigating the generation rate of and the ion energies in these turbulent inter-ELM filaments using the same approach as in Ref. [49] for the L-mode discharges in AUG would be a good subject for future work.

The procedure illustrated in Fig. 10 is applied for all plasma pulses from Table 1. The resulting $T_{i E L M}$ is plotted against $\Delta r_{\text {sep }}, W_{E L M}$ and $W_{E L M} / W_{\text {plasma }}$ in Fig. 12. The error bars on $\Delta r_{\text {sep }}$ account for the typical uncertainty of $5 \mathrm{~mm}$ in the probe-separatrix distance. In the far SOL, $T_{i E L M}$ is in the range $20-200 \mathrm{eV}$, which is $5-50 \%$ if the ion temperature at the pedestal top. As can be seen from Fig. 12, $T_{i E L M}$ decreases with $\Delta r_{\text {sep }}$ (e-folding length $\lambda_{T i} \approx 10 \mathrm{~mm}$ in the far SOL for $W_{E L M} \approx 27-35 \mathrm{~kJ}$ ) and increases with $W_{E L M}$. Note that $T_{i E L M}$ at $\Delta r_{\text {sep }}=58 \mathrm{~mm}$ has been measured in the discharge with the OL shadow shifted by 5$12 \mathrm{~mm}$ outward. This was achieved by shifting both, the outboard midplane separatrix and RFA target position, inward. As a result $L_{/ /}$at $\Delta r_{\text {sep }}=58 \mathrm{~mm}$ is longer and, therefore, the parallel energy dissipation in the ELM filaments can be somewhat weaker compared with other measurements at $W_{E L M} \approx 35 \mathrm{~kJ}$. This might explain the "flattening" of $T_{i E L M}$ profile for $\Delta W_{E L M} \approx 35 \mathrm{~kJ}$. Because of a different magnetic equilibrium in the discharge in question, the measurement of $T_{i E L M}$ at $\Delta r_{\text {sep }}=58 \mathrm{~mm}$ is excluded from the fit of $\lambda_{T i}$.
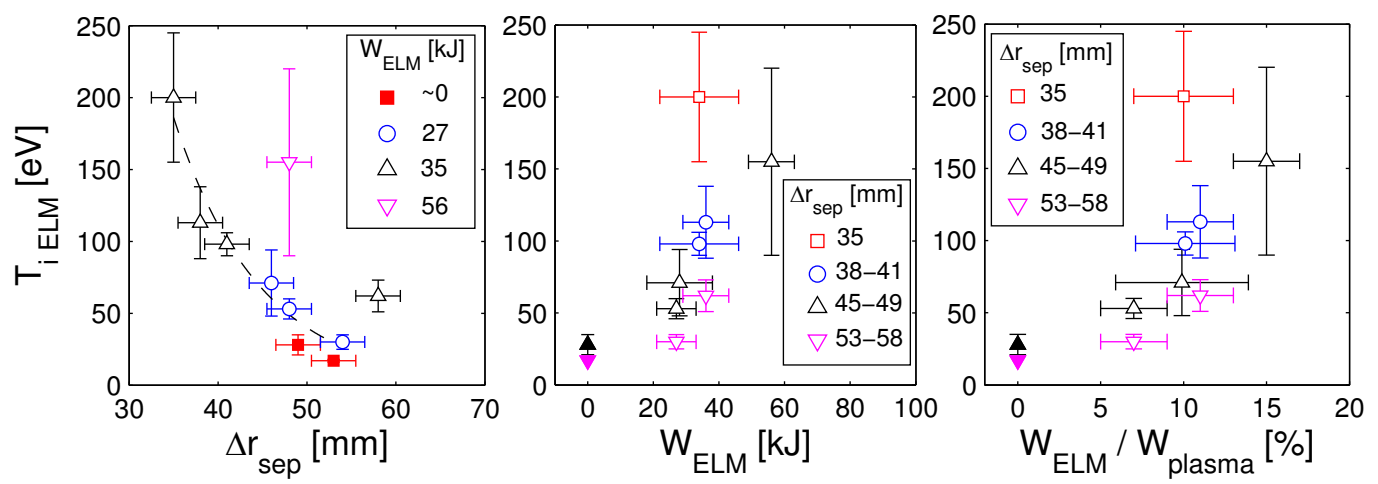

Figure 12. Dependence of $T_{i E L M}$ on $\Delta r_{\text {sep }}$ and the energy lost per ELM (absolute and relative to $\left.W_{\text {plasma }}\right)$. Dashed line is the exponential fit to $T_{i E L M}$ measured for $W_{E L M} \approx 27$ and $35 \mathrm{~kJ}$ with the e-folding length $\lambda_{T i} \approx 10 \mathrm{~mm}$ (for the reason described in the text, the measurement of $T_{i E L M}$ at $\Delta r_{\text {sep }}=58 \mathrm{~mm}$ is excluded from the fit). Color coding is preserved in the following figures. 
The decrease of $T_{i E L M}$ with increasing $\Delta r_{\text {sep }}$ is easily explained by the energy losses along the magnetic field lines to the solid surface as the ELM filaments propagate across the SOL. Strong dependence of $T_{i E L M}$ on $W_{E L M}$ might have two possible interpretations: filaments of larger ELMs (i) are spilled into the SOL with higher initial temperatures so that they simply arrive hotter into the far SOL or (ii) they propagate faster radially and have less time to lose their energy along the field lines (or (iii) the combination of both).

Interpretation (i) is highly unlikely, because the variation of $T_{i p e d}$ is too small to account for the observed dependence of $T_{i E L M}$ on $W_{E L M}$ (Table 1). Moreover, the filaments with higher initial temperatures are also subject to stronger parallel energy loss since the characteristic parallel transport time $L_{/ /} / c_{s} \propto L_{/ /} / \sqrt{T_{i}+T_{e}}$. Therefore, large variation of the initial ELM filament ion temperature should actually result in small changes in the far SOL $T_{i E L M}$. For the same reason, $T_{i E L M}$ would vary only little if the filaments were coming from different positions (and thus different initial temperatures) within the pedestal, as will be demonstrated in Sec. 6.

Eq. (1) can be used to estimate the approximate average radial propagation speed of ELM filaments, $v_{r}$, from the RFA measurements. The values of $\lambda_{j s a t}$ and $T_{i E L M}$ are respectively from Figs. 9 and 12. It can be assumed that $T_{i E L M}>T_{e E L M}$ (e.g. $[10,17,33,50]$ and Sec. 6) so that $c_{s}$ can be approximated by the ELM ion thermal speed $\sqrt{e T_{i E L M} / m_{i}}$. In Eq. (1), $c_{S} / L_{/ /}$represents the particle sink rate of a filament along the flux tube connected at each end to a solid surface. Therefore, $L_{/ /}$is evaluated as one half of the harmonic mean of the lengths of the field lines between the outer midplane and the nearest surface for both directions along the field lines, i.e.

$$
\left\langle L_{/ /}\right\rangle=L_{/ /}^{\prime} L_{/ /}^{\prime \prime} /\left(L_{/ /}^{\prime}+L_{/ /}^{\prime \prime}\right)
$$

The values of $L_{/ /}^{\prime}$ and $L_{/ /}^{\prime \prime}$ are obtained from the magnetic field lines tracing. Fig. 13 shows $\left\langle L_{/ /}\right\rangle$as a function of $\Delta r_{s e p}$ evaluated for the field lines passing the RFA. It was verified by making a $5^{\circ}$ scan of $\left\langle L_{/ /}\right\rangle$as a function of the toroidal angle that the effect of the stochastic fields due to magnetic perturbations on $\left\langle L_{/ /}\right\rangle$in the discharge series \#6 does not need to be 
considered. As can be seen from Fig. 13, $\left\langle L_{/ /}\right\rangle$is at most a few meters for $\Delta r_{\text {sep }} \approx 35-60 \mathrm{~mm}$ where the present RFA measurements have been obtained. Two effects might influence the effective $\left\langle L_{/ /}\right\rangle$. First, in the frame of a toroidally rotating filament $\left\langle L_{/ /}\right\rangle$can vary due to toroidally discrete OLs in AUG. To account for this effect one would need to perform for each equilibrium a computationally demanding calculation of the toroidally averaged $\left\langle L_{/ /}\right\rangle$. Second, in Eq.(2) the filament is viewed as a packet located at a single position along the flux tube, namely the one corresponding to the outer midplane. However, as routinely observed by the fast visible cameras (e.g. $[6,48,51])$ and seen clearly from Fig. 6 , the filaments extend over long parallel distances as they propagate outwards. This, in turn, might influence the effective filament $\left\langle L_{/ /}\right\rangle$given that some parts of the filament are closer to the end of the flux tube. In view of these uncertainties, we assume in Eq.(1) $\left\langle L_{/ /}\right\rangle=1 \mathrm{~m}$ for simplicity.

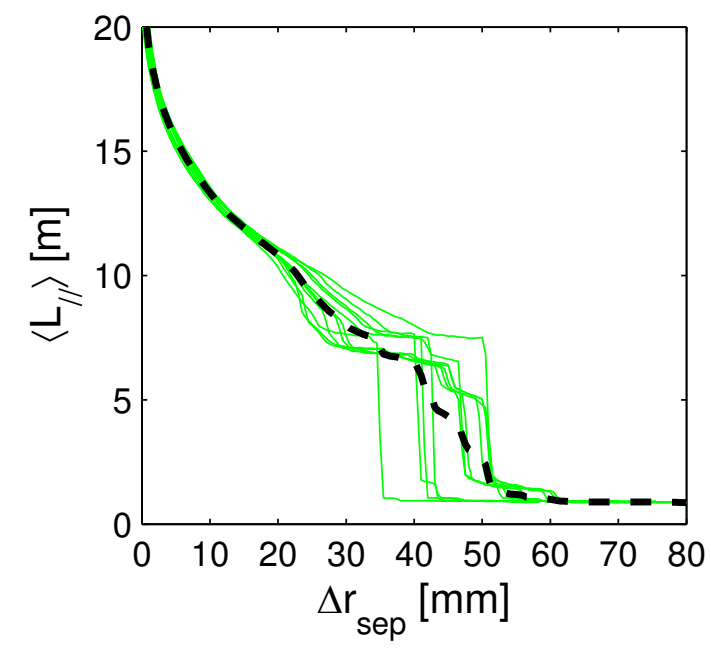

Figure 13. Parallel connection length given by Eq. (2) for individual discharges from Table 1. Dashed line: average $\left\langle L_{/ /}\right\rangle$for all plasma pulses.

In Fig. 14, $v_{r}$ evaluated from Eq.(1) is plotted against $W_{E L M}$. The error bars account for uncertainties in $\lambda_{j s a t}$ and $T_{i E L M}$, and are large as is common with any $v_{r}$ measurements. $v_{r} \approx 400-2500 \mathrm{~m} / \mathrm{s}$ which, given the uncertainty in $\left\langle L_{/ /}\right\rangle$, is in a surprisingly good agreement with earlier measurements of $v_{r}$ in the AUG far SOL $[24,25] . v_{r}$ tends to increase with $W_{E L M}$, suggesting that on average the filaments in large ELMs might propagate faster 
radially and have less time to cool before reaching the far SOL. The increase of $v_{r}$ with $W_{E L M}$ could explain the inverse scaling of the JET divertor ELM energy fraction $[19,50]$ as well as the increase of the JET limiter ELM loading with $W_{E L M}[5,20]$ (in [22], however, the energy deposited on the main wall limiters in JET by ELMs was found to be independent of $\left.W_{E L M}\right) . v_{r}$ seems to be roughly independent of $\Delta r_{\text {sep }}$, at least for the range of radii covered by RFA and within the uncertainty of the measurements.

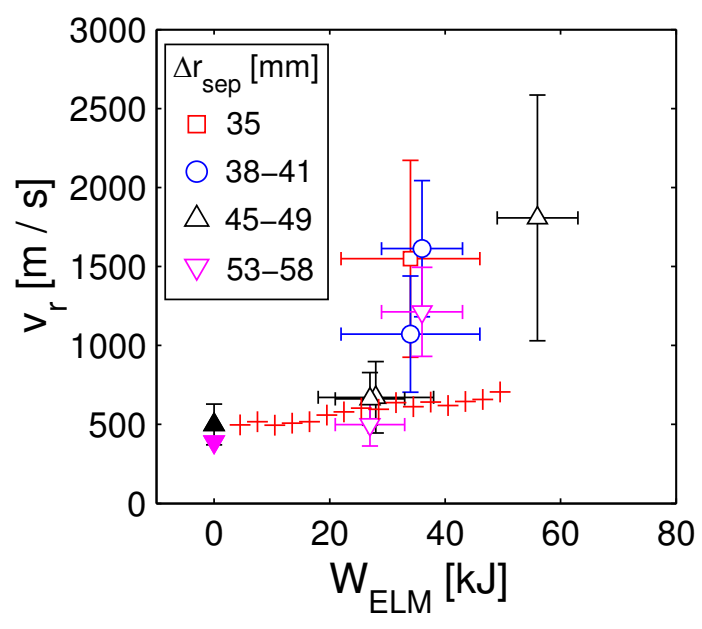

Figure 14. Dependence of the ELM-averaged radial filament propagation speed estimated from the RFA measurements on the energy lost per ELM. Also plotted is $v_{r}$ obtained from the measurements of the poloidal electric field (+) [24].

A note is required regarding the comparison with earlier measurements of $v_{r}$ in Type I ELMs in the AUG far SOL $[24,25]$. In earlier experiments $v_{r}$ was obtained in individual ELM filaments from the time difference in the signals between two separated probes [25], and from the probe measurements of the poloidal electric field [24]. This is in contrast to the present ELM-averaged quantities which are determined by the most intense filaments of a given ELM and thus might be more relevant when addressing the issues of the ELM-wall interaction. In order to allow for consistent comparison with the present experiment, the values of $v_{r}$ obtained earlier in the AUG far SOL [24] were averaged over the six first filaments of each ELM with the negative values of $v_{r}$ excluded. As can be seen in Fig. 14, average radial filament propagation speed obtained in this way shows a dependence on $W_{E L M}$, albeit much weaker than that estimated from the RFA measurements. 


\section{Thermography-RFA comparison}

Measurements of the heat flux on the leading edge of the RFA housing by a twodimensional IR camera are available for discharge \#26164. The camera has a $56 \times 24$ pixel array, integration time of $5.4 \mu$ s and time resolution of $80 \mu \mathrm{s} /$ frame. As shown in Fig. 15, the camera views the $\mathrm{i}$-side RFA which is aligned within $<1^{\circ}$ with the magnetic field lines. The vertical axis of the pixel array is approximately tangential to the local flux surfaces. The IRinferred heat flux density, $q^{I R}$ is calculated from the jitter-corrected temperature measurements using the THEODOR code [52].

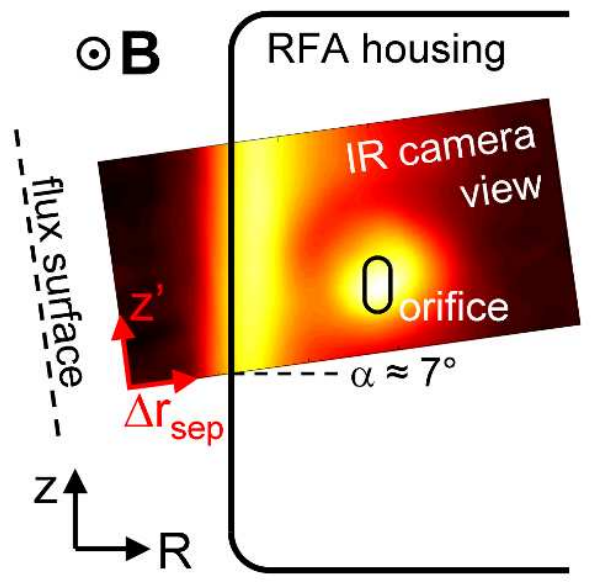

Figure 15. The IR camera view of the RFA housing. Machine coordinates $(R, z)$ are tilted by $\sim 7^{\circ}$ with respect to the frame coordinates $\left(r, z^{\prime}\right) . z^{\prime}$ is approximately tangential to the local flux surfaces.

Typical camera images taken during and just after a Type I ELM $\left(W_{E L M}=50 \mathrm{~kJ}\right)$ are illustrated in Fig. 16. The blurred edge of the probe housing results from the imperfections in the camera focusing combined with the edge chamfering (to prevent excessive thermal loading). The bright spot observed during an ELM at $\Delta r_{\text {sep }} \approx 48 \mathrm{~mm}$ is the orifice in the housing, behind which the RFA slit plate is located (Fig. 2), and at which $q^{I R}$ is falsely high because of a different materials involved. The horizontal dashed line indicates the region along which the e-folding length $\lambda_{q}$ is obtained from the exponential fit to the parallel heat flux density $q_{\|}^{I R}$ plotted against $\Delta r_{\text {sep }}$, as illustrated in Fig. 16, right. During an ELM $q_{\|}^{I R}$ is a factor of 2-4 higher and the heat flux density profile flattens compared to the inter-ELM period, which can be related to enhanced radial transport of particles and energy during an ELM. 
In the far SOL, the length of the field lines between the OL and the RFA is a factor of $\sim 10$ shorter compared with $L_{/ /}$between RFA and the outer divertor. Because of the short parallel distance between the RFA and the OL, the length of the field lines between the RFA and the outer divertor is almost the same as the one between the OL and the outer divertor in the absence of the probe. Note also that the effective disturbance length [53] of both, the OL and the RFA (up to several tens of meters), is longer than $L_{/ /}$between the OL and the outer divertor (up to 10 meters, Fig. 8). Therefore, $\lambda_{q}$ monitored along the probe head is likely the same as the one that would be measured along the OL in the absence of the probe.
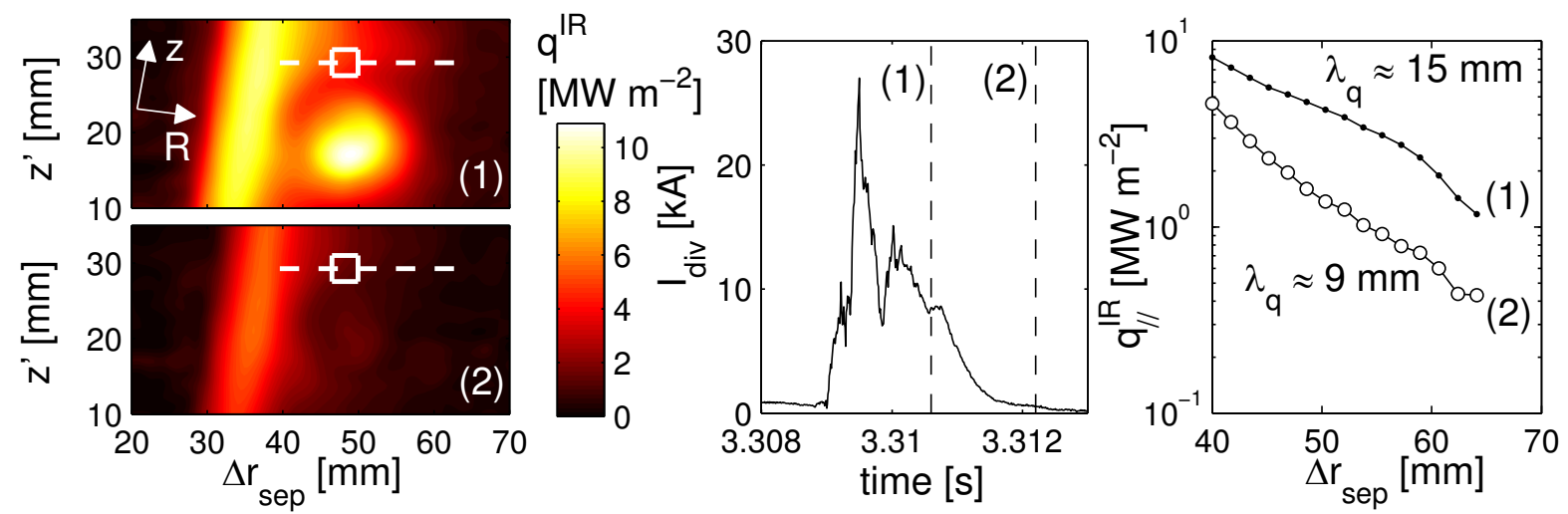

Figure 16. Left: IR camera images of $q^{I R}$ during (1) and just after (2) a Type I ELM $\left(W_{E L M}=50 \mathrm{~kJ}\right)$ as indicated on the time trace of $I_{d i v}$ (middle panel). The bright spot seen in the top image is the orifice of the RFA housing. The white square indicates the ROI from which $q^{I R}$ is taken in figures 17 and 18. The horizontal dashed line indicates the region along which the radial profiles of the parallel heat flux density (right panel) are read and the corresponding e-folding lengths $\lambda_{q}$ obtained.

In Fig. 17, $q_{/ /}^{I R}$ at the ROI at $\Delta r_{\text {sep }} \approx 48 \mathrm{~mm}$ (highlighted in Fig. 16) is compared with the parallel heat flux density $q_{/ /}^{R F A}$ estimated from the RFA measurements obtained at similar separatrix distance. It is well known that such thermography-probe comparison is a complex task because of the lack of knowledge of many parameters needed to evaluate absolutely calibrated parallel heat flux density from the probe data $([54,55]$ and references therein). Nevertheless, we evaluate $q_{/ /}^{R F A}$ in the usual way as 


$$
q_{\|}^{R F A}=\gamma j_{s a t} T_{e E L M}
$$

with the heat transmission coefficient $\gamma$ given by Eq. (25.46) in [56]:

$$
\gamma=2.5 \frac{T_{i E L M}}{T_{e E L M}}+2-0.5 \ln \left[2 \pi \frac{m_{e}}{m_{i}}\left(1+\frac{T_{i E L M}}{T_{e E L M}}\right)\right] .
$$
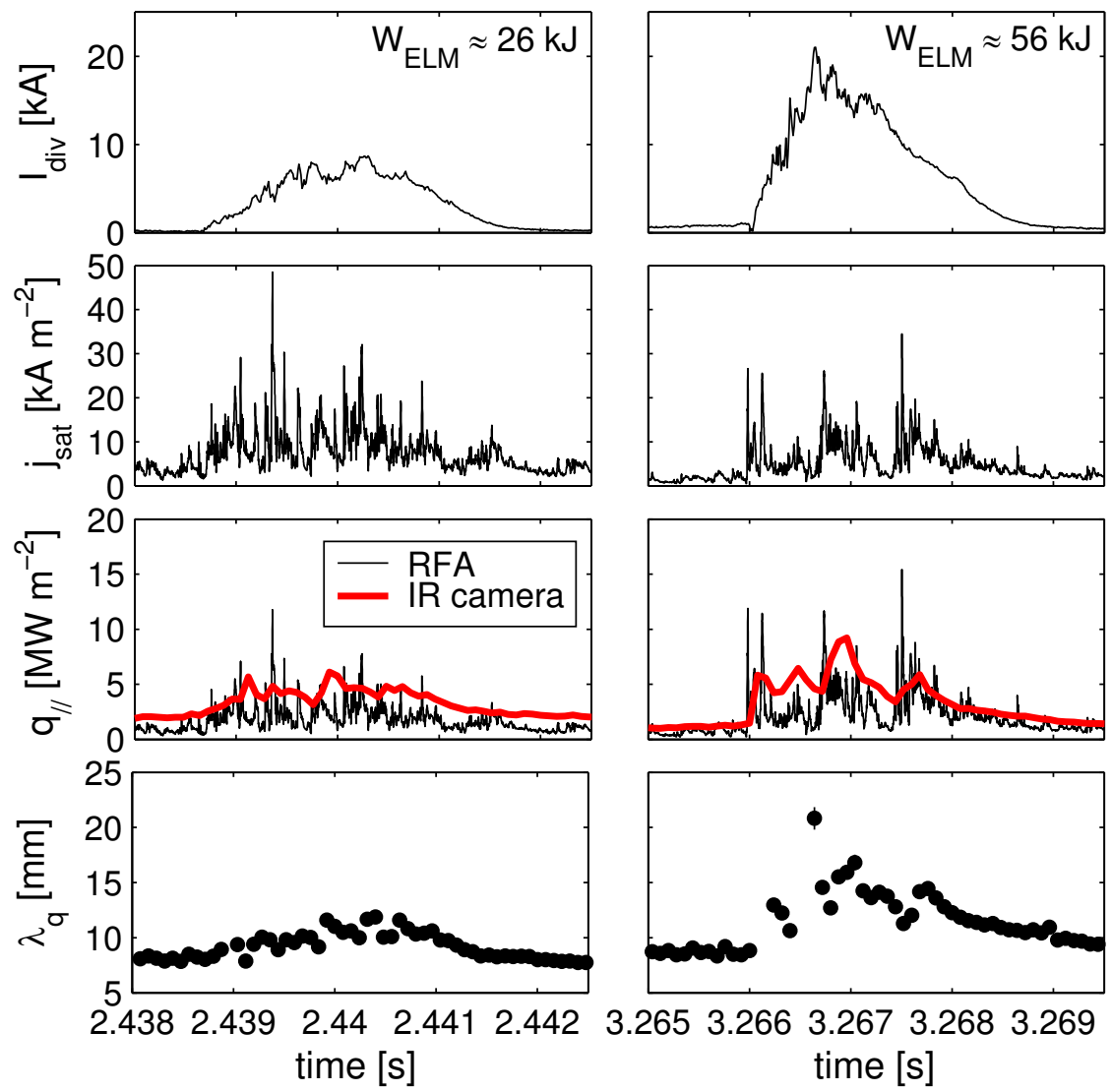

Figure 17. Evolution of the IR-camera and RFA signals during two Type I ELMs characterized by different $W_{E L M}$. From top to bottom: $I_{\text {div }}, j_{\text {sat }}$ measured at $\Delta r_{\text {sep }} \approx 46-48$ mm, parallel heat flux densities measured by the IR camera at the ROI indicated in Fig. 16 and estimated from the RFA measurements of $j_{\text {sat }}$ and $T_{i E L M}$ (Eq. 3). Bottom panels: efolding length of $q_{/ /}^{I R}$ obtained from the thermographic measurements along the ROI indicated by dashed line in Fig. 16, left. 
In Eq. (4) it is assumed that the net current through the sheath is zero and the secondary electron emission from the slit plate can be neglected, so that the equation gives the lower estimate of $\gamma$. The values $T_{i E L M}=71 \mathrm{eV}$ (for $W_{E L M}=26 \mathrm{~kJ}$ ) and $T_{i E L M}=155 \mathrm{eV}$ $\left(W_{E L M}=56 \mathrm{~kJ}\right)$ are taken from the RFA measurements at $\Delta r_{s e p} \approx 46-48 \mathrm{~mm}$, described in Sec. 4.2. We assume $T_{e E L M}=15 \mathrm{eV}$ which corresponds approximately to the ELM-averaged electron temperature observed previously in the AUG far SOL [31], albeit for different plasma parameters. Equation (4) gives $\gamma \approx 16$ for $W_{E L M}=26 \mathrm{~kJ}$ and $\gamma \approx 30$ for $W_{E L M}=56 \mathrm{~kJ}$.

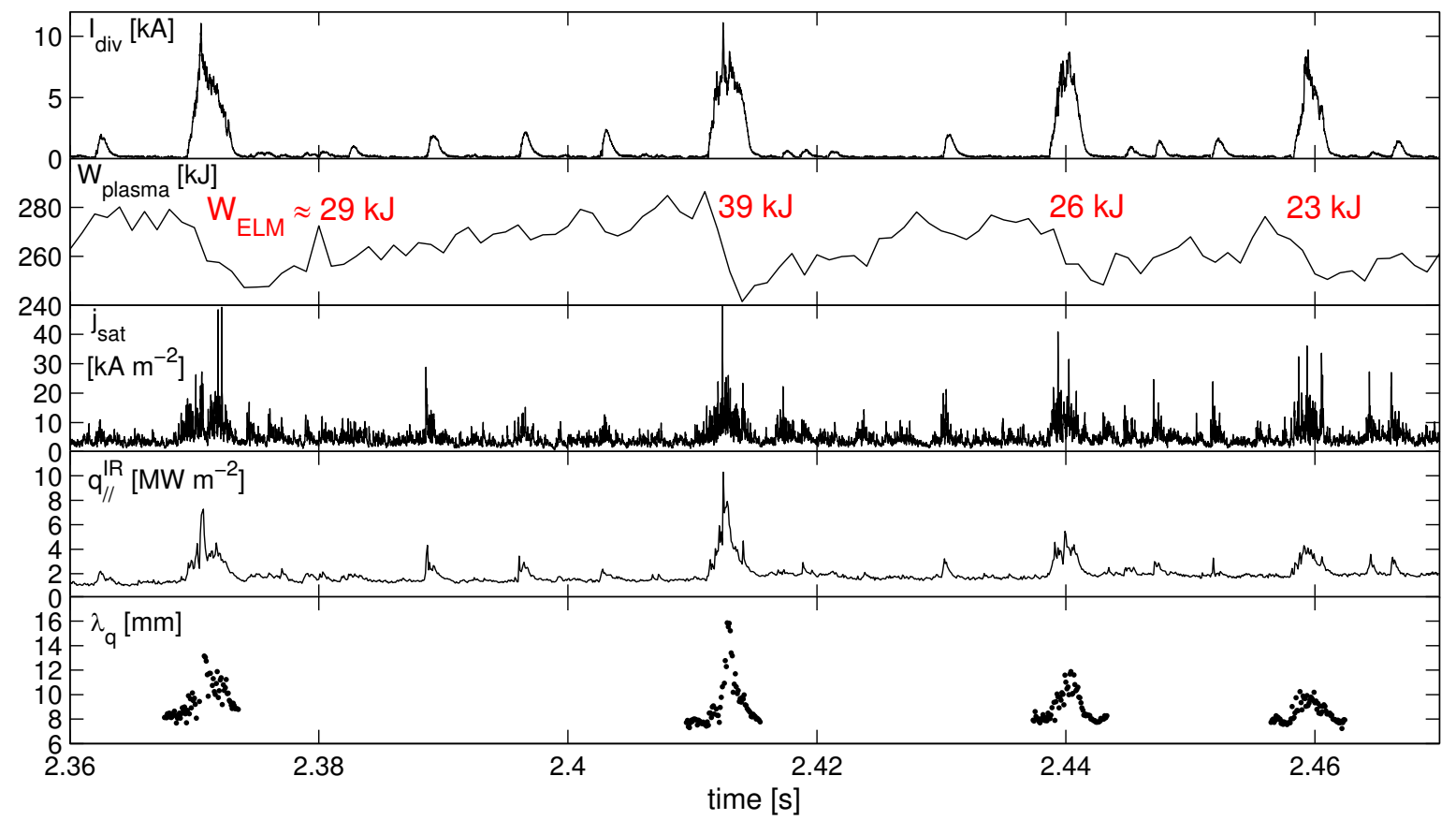

Figure 18. From top to bottom: time dependence $I_{\text {div }}, W_{\text {plasma }}, j_{\text {sat }}$ and $q_{/ /}^{I R}$ (measured at $\Delta r_{\text {sep }} \approx 46 \mathrm{~mm}$ in the discharge \#26164) and $\lambda_{q}$ along the RFA housing. The signals comprise four Type I ELMs each with different $W_{E L M}$.

As seen from Fig. 17, $q_{/ /}^{I R}$ is up to a factor of 2 higher compared to $q_{/ /}^{R F A}$, which is a fairly good agreement in view of simplifying assumptions in equations (3) and (4). Additionally, we have not taken into account the attenuation of the incident ion current on the RFA housing due to ion deflection in the magnetic pre-sheath [57]. This effect might reduce the collecting area of the slit plate and lead underestimation of $j_{\text {sat }}$ measured by the RFA by a factor of $\sim 2.5$. If this effect was taken into account, $q_{/ /}^{R F A}$ would agree better with $q_{/ /}^{I R}$. In the 
smaller of the two ELMs plotted in Fig. 17, the duration of the filaments is too short compared to the IR camera time resolution. On the other hand, for the larger ELM, which seems to contain fewer but more intense current bursts, the largest filaments are somewhat better resolved by the IR camera.

Independently of the RFA measurements of the ELM ion energy in Sec. 4.2, two important pieces of information about far SOL ELM ion energies can be extracted from the comparison of $j_{\text {sat }}$ and $q_{/ /}^{I R}$. First, if the RFA measurements of $T_{i E L M}$ were not taken into account and $T_{i E L M}=T_{e E L M}=15 \mathrm{eV}$ was assumed in equations (3) and (4), the computed $q_{/ /}^{R F A}$ would be up to an order of magnitude lower than $q_{/ /}^{I R}$. This indicates that ELM ions reach the far SOL with relatively large energies, consistent with the observations from sections 3.2 and 4.2. Second, $q_{/ /}^{I R}$ observed during the ELM increases by up to $\sim 50 \%$ as $W_{E L M}$ increases from 26 to $56 \mathrm{~kJ}$. The same observation is obtained from Fig. 18. Curiously, while $q_{/ /}^{I R}$ increases with ELM size, $j_{s a t}$ seems to be independent of $W_{E L M}$, as already observed in Fig. 8 and seen also in figures 17 and 18 . The fact that $q_{/ /}$increases with $W_{E L M}$ whilst $j_{\text {sat }}$ does not indicates that the characteristic ELM ion energy increases with $W_{E L M}$ which is again consistent with the observations from Sec. 4.2 .

The bottom panels in Fig. 17 show the time evolution of $\lambda_{q}$ obtained from $q_{/ /}^{I R}$ in the same way as in Fig. 16. As already seen in Fig. 16, $\lambda_{q}$ increases during an ELM compared to the inter-ELM period. Fig. 18 shows the values of $\lambda_{q}$ measured in a single time sequence which features four Type I ELMs with different $W_{E L M}=23-39 \mathrm{~kJ}$. The values of $\lambda_{q}$ assembled from figures 17 and 18 , sampled $\pm 0.5 \mathrm{~ms}$ around the maximum $q_{/ /}^{I R}$ for each ELM, are plotted against $W_{E L M}$ in Fig. 19. In order to look for trends, the mean value and the standard deviation of $\lambda_{q}$ from each ELM are also shown. $\lambda_{q}$ tends to increase with $W_{E L M}$, similar to $\lambda_{\text {jsat }}$ from Fig. 9, though the trend is marginal within the scatter of the data. Nevertheless, recalling a balance between the characteristic parallel and perpendicular ELM transport times in the SOL from Sec. 4.1,

$$
\frac{v_{r}^{(1)}}{v_{r}^{(2)}} \approx \frac{\lambda_{q}^{(1)}}{\lambda_{q}^{(2)}} \sqrt{\frac{T_{i E L M}^{(1)}}{T_{i E L M}^{(2)}}}
$$


as well as a relatively strong increase of $T_{i E L M}$ with $W_{E L M}$, Fig. 19 suggests that $v_{r}$ increases with $W_{E L M}$, consistent with the observation from Fig. 14.

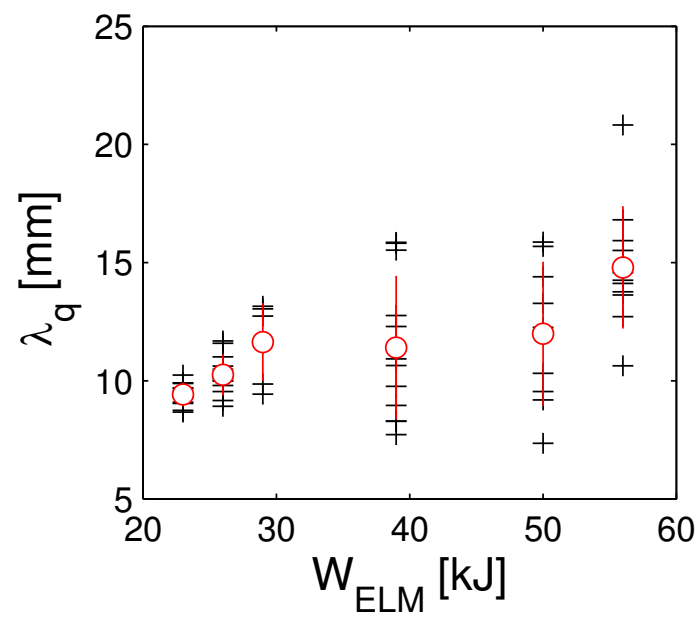

Figure 19. ELM heat flux density e-folding length measured along the RFA housing by the fast IR camera plotted against the energy lost per ELM. Also plotted is the mean and the standard deviation for each ELM.

The values of $\lambda_{q}$ from Fig. 19 can be compared to $\lambda_{\text {jsat }}$ from Fig. 9 measured during similar ELMs. The RFA measures $\lambda_{j s a t}=11 \pm 1 \mathrm{~mm}$ for $W_{E L M}=26 \mathrm{~kJ}$ and $\lambda_{j s a t}=21 \pm 2 \mathrm{~mm}$ $W_{E L M}=56 \mathrm{~kJ}$, i.e. $\lambda_{j s a t} \approx(1-1.5) \lambda_{q}$. Although the measurements suggest that $\lambda_{j s a t} / \lambda_{q}$ might increase with $\Delta W_{E L M}$, given the sparseness of the simultaneous measurements of $\lambda_{j s a t}$ and $\lambda_{q}$, one cannot make reliable conclusions about the evolution of $\lambda_{j s a t} / \lambda_{q}$ with the ELM size. Nonetheless, a similar relation between the heat flux density and the ion current e-folding lengths was previously observed in the AUG far SOL in [30]. Comparable values of $\lambda_{j s a t}$ and $\lambda_{q}$ was taken as an indication that the convection dominates the far SOL ELM heat transport.

\section{6. $v_{r}$ : comparison with the parallel loss model}

Previous sections suggest that the measured $v_{r}$ tends to increase with $W_{E L M}$. This evidence is reconciled here with the predictions of a simple fluid model of the parallel ELM transport developed in [33] and used to interpret earlier RFA and IR camera observations in JET $[4,5,22,33,49,58]$ and AUG [10]. In the model, the ELM filament transport is described in the filament frame of reference by the temporal evolution of a Gaussian wave packet in which the initial particle and energy content decreases due to parallel losses to the 
divertor targets $\left(\propto c_{s} /\left\langle L_{/ /}\right\rangle\right)$. Ion and electron fluids are coupled through the equipartition term. $v_{r}$, an arbitrary input parameter, relates the time of the filament propagation and the radial distance from the filament birth location. The latter can be assumed to be at the pedestal top, i.e. $\Delta r_{\text {sep }}=-15 \mathrm{~mm}$ in the discharges described here. The values of $T_{i p e d}$ and $n_{\text {eped }}$ from Tab. 1 determine the filament initial temperatures and density, assuming $T_{\text {eped }}=T_{i \text { ped }}$ for simplicity. Although this is not strictly true (see Table 1), thermal coupling of ions and electrons has almost no effect on filament $T_{i E L M}$. Moreover, as will be shown in Fig. 23, the filament temperatures in the far SOL predicted by the model are only weakly correlated with those at the filament birth location, meaning that this assumption does not affect strongly far SOL $T_{e E L M}$ obtained from the model. $\left\langle L_{/ /}\right\rangle$is given by the average connection length profile from Fig. 13. Note that the energies with which the ELM filaments arrive in the far SOL are determined by the filament evolution first few $\mathrm{cm}$ outside the separatrix, where $\left\langle L_{/ /}\right\rangle$is very similar for all discharges. For each measured $T_{i E L M}, v_{r}$ is adjusted to bring the simulated and the measured $T_{i E L M}$ into agreement, as illustrated in Fig. 20. In Fig. 20 the filament is launched with $T_{i p e d}=T_{\text {eped }}=350 \mathrm{eV}$ and $n_{\text {eped }}=7.7 \times 10^{19} \mathrm{~m}^{-3}$. The error bar in $v_{r}$ is obtained by matching the confidence interval of the measured $T_{i E L M}$. The general trend $T_{i E L M}>T_{e E L M}$ observed in the simulations can be explained by higher parallel conductivity of electrons compared to that of ions, and agrees with earlier experimental observations [10, 31] as well as kinetic ELM simulations [50]. Also shown in Fig. 20 are the characteristic radial decay lengths of ion and electron temperatures, $\ell_{T i, e}=-\left[\left(d T_{i, e} / d r\right) / T_{i, e}\right]^{-1}$ as a function of $\Delta r_{\text {sep }}$. In the far SOL, $\ell_{T i}$ is on average a factor of 2 higher compared with $\lambda_{T i} \approx 10 \mathrm{~mm}$ estimated from Fig. 12. 


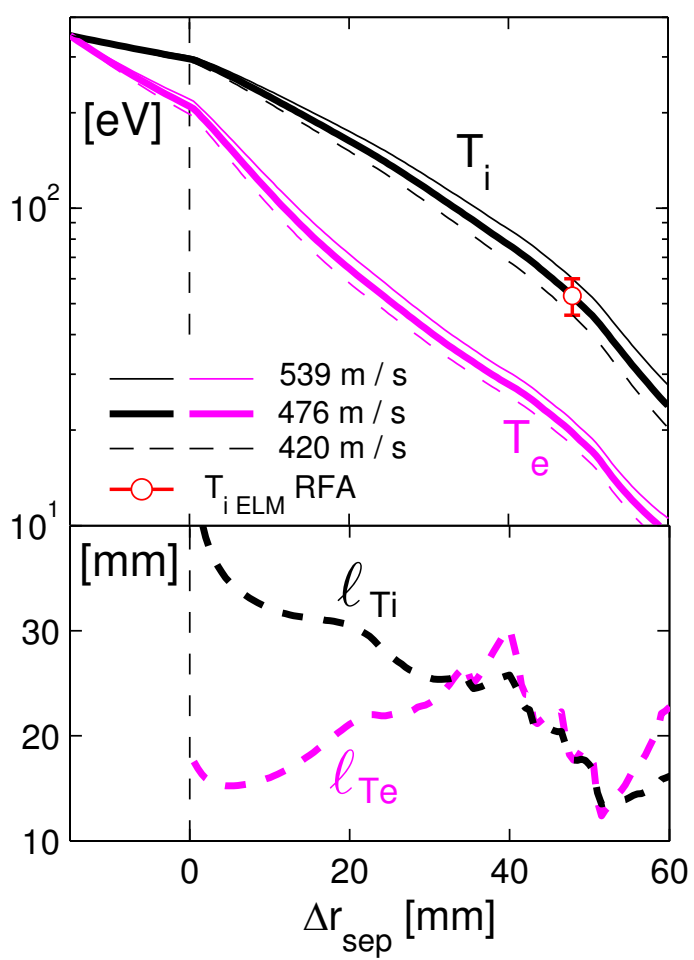

Figure 20. Top: Filament ion and electron temperatures as a function of $\Delta r_{\text {sep }}$ obtained from the parallel loss model. The radial filament propagation speeds $v_{r}$ are adjusted to match $T_{i E L M}=53 \pm 7 \mathrm{eV}$ measured by the RFA at $\Delta r_{\text {sep }}=48 \mathrm{~mm}$. Bottom: characteristic radial decay lengths of ion and electron temperatures.

In Fig. 21, $v_{r}$ obtained from the model are plotted against $W_{E L M}$ using the same color code for $\Delta r_{\text {sep }}$ as in Fig. 14. In agreement with the RFA measurements, the predicted $v_{r}$ tends to increase with $W_{E L M}$, though the trend should be treated with some caution because of large uncertainties. Unconstrained non-linear least-squares fit gives $v_{r} \propto W_{E L M}^{1.50 \pm 0.39}$ which is stronger dependence compared with the interchange scaling $\left(v_{r} \propto W_{E L M}^{1 / 2}\right)$. Surprisingly (in view of the applied approximations and uncertainties), as seen from Fig. 22, the range of $v_{r}$ predicted by the model is in good agreement with $v_{r}$ estimated from RFA measurements. 


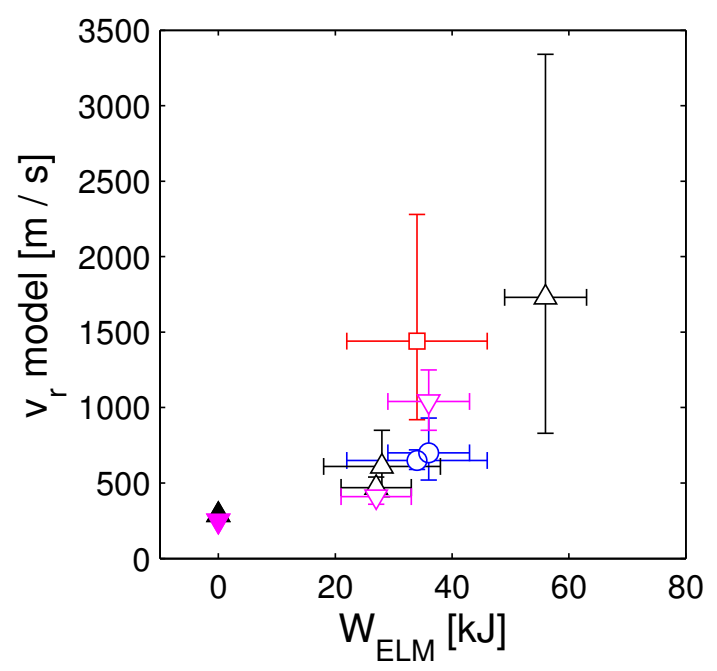

Figure 21. Radial ELM filament propagation speed required in the parallel filament loss model to bring simulated $T_{i E L M}$ into agreement with RFA measurements. The filaments are launched from the pedestal top.

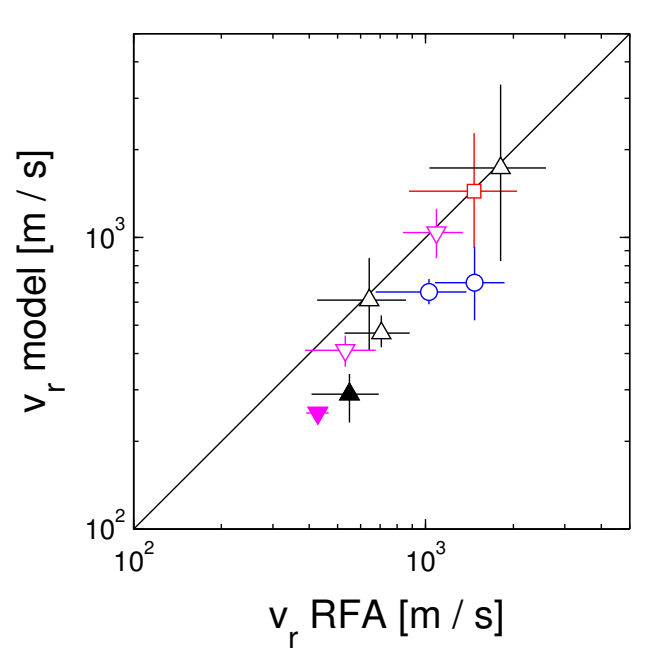

Figure 22. Radial ELM filament propagation speed from Fig. 21 plotted against $v_{r}$ estimated from the RFA measurements.

For reasons mentioned in Sec. 4.2 and as illustrated in Fig. 23, the filament temperatures in the far SOL predicted from the simulations are only weakly sensitive to the filament birth location (which translates into the filament initial temperatures and density). In Fig. 23, the filament temperatures predicted from Fig. 20 are compared with those obtained by launching the filament from the separatrix $\left(n_{\text {esep }} \cong 2 \times 10^{19} \mathrm{~m}^{-3}\right.$ from the lithium beam diagnostic, $T_{i s e p} \cong 200$ from CXRS) assuming $v_{r}=476 \mathrm{~m} / \mathrm{s}$ as in Fig. 20. Fig. 23 shows that different assumptions about the initial filament conditions leads to a slight $(\sim 10 \mathrm{eV})$ but not decisive difference in the predicted filament temperatures in the far SOL. Therefore, the values of $v_{r}$ similar to those in Fig. 21 would be obtained if the filaments were launched from different positions between the pedestal top and the separatrix. This is very practical result, given the uncertainty in the position at which the ELM filaments observed in the SOL originate. Additionally, it is worth noticing that $v_{r}$ would vary at most by $11 \%$ if all simulations were reproduced for fixed $T_{i \text { ped }}=T_{e \text { ped }}=350 \mathrm{eV}$. On the other hand, as shown in 
Fig. 24, the pedestal temperatures required to match $T_{i E L M}$ would disagree with the spectroscopic measurements if all simulations were reproduced assuming constant $v_{r}=500$, 1000 or $1500 \mathrm{~m} \mathrm{~s}^{-1}$ (note that the values $T_{i p e d}>1 \mathrm{keV}$ obtained from the simulations for intermediate and large ELMs assuming $v_{r}=500$ and $1000 \mathrm{~m} / \mathrm{s}$ are not shown in Fig. 24). Again, this is due to the coupling of the local filament temperature and the parallel losses which makes $T_{i E L M}$ in the far SOL only weakly sensitive to the initial filament ion temperature.

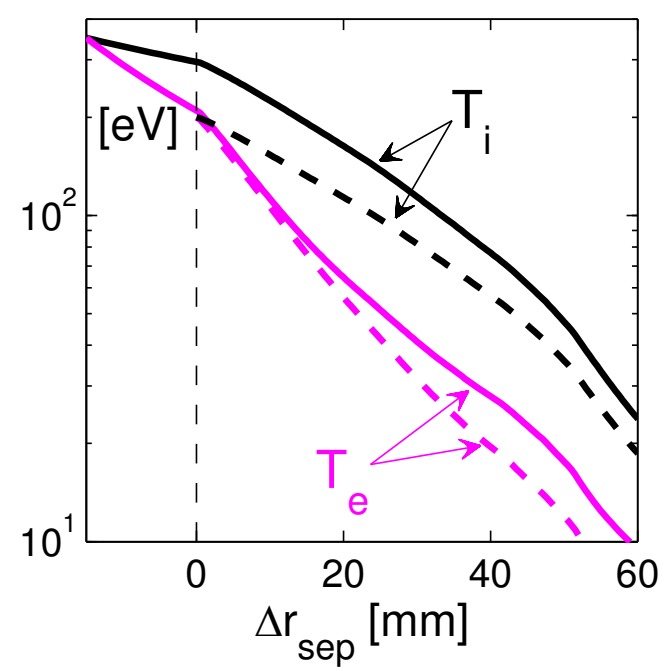

Figure 23. Filament ion and electron temperatures as a function of $\Delta r_{\text {sep }}$, obtained from the parallel loss model assuming $v_{r}=476 \mathrm{~m} / \mathrm{s}$. ELM filament is launched from the pedestal top (full) and from the separatrix (dashed) with the corresponding temperatures and electron density.

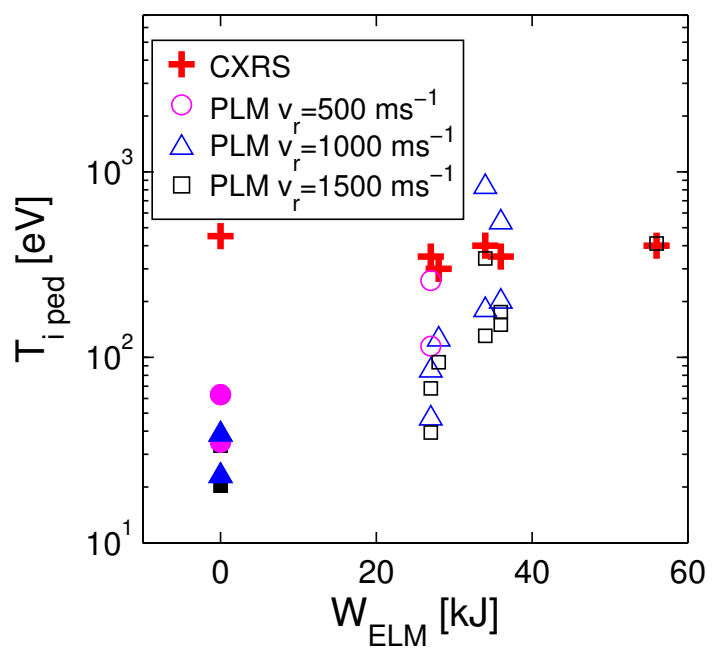

Figure 24. Pedestal temperatures required in the filament loss model to match the measured $T_{i E L M}$, assuming $v_{r}=500,1000$ and 1500 $\mathrm{m} / \mathrm{s}$ in all simulations. Note that $T_{i p e d}>1 \mathrm{keV}$ from the simulations are not shown. Also plotted is $T_{i p e d}$ measured by the edge CXRS diagnostic. 


\section{Summary and conclusions}

The thermal load during ELMs is recognized as a concern for the ITER first wall lifetime and has been addressed in a number of studies. New measurements of the ion energies and current in Type I and, for the first time, in mitigated Type I ELMs, were carried out in the AUG SOL using an RFA. ELM-averaged ion temperatures $T_{i E L M} \approx 20-200 \mathrm{eV}$, i.e. $5-50 \%$ of the pedestal top ion temperature, are observed in the far SOL, $35-60 \mathrm{~mm}$ outside the separatrix, corresponding to $15-25 \mathrm{~mm}$ in front of the outboard limiter. $T_{i E L M}$ in this region decreases with the separatrix distance with an e-folding length of $\sim 10 \mathrm{~mm}$ and increases with the ELM energy $W_{E L M}$. The lowest $T_{i E L M}$ is measured during mitigated ELMs. The increase of $W_{E L M}$ is associated with the flattening of the radial profile of the ELMaveraged ion current density in Type I ELMs. RFA observations, substantiated by the fast IR camera measurements, as well as the predictions of a simple fluid model of parallel ELM transport, indicate that on average the filaments of large ELMs propagate faster radially. RFA data suggest a somewhat stronger than linear dependence of the ELM-averaged radial filament propagation speed $v_{r}$ on $W_{E L M}$, though the experimental uncertainties are large. Nevertheless, the trend is consistent with earlier thermographic measurements (i.e. the fraction of $W_{E L M}$ deposited outside the divertor increases with the ELM energy) [2, 5, 19-21].

\section{Acknowledgements}

This project was supported in part by the Association Euratom-CEA. We wish to thank to R. Neu and the two anonymous referees for helpful comments. In this work we used the script of the parallel loss model written by Dr. D. Moulton. We also would like to thank Andranik Sarkissian and Guy Lebrun for useful discussions about the RFA design and Plasmionique Inc. (Canada) for the high quality with which they manufactured the RFA probe.

\section{References}

[1] A. Herrmann, T. Eich, C. J. Fuchs, J. Neuhauser and ASDEX Upgrade Team, Plasma Phys. Control. Fusion 46 (2004) 971.

[2] A. Loarte et al., Phys. Plasmas 11 (2004) 2668. 
[3] N. Asakura, M. Takeschi, N. Oyama and T. Nakano, J. Nucl. Mater. 337-339 (2005) 712 .

[4] R. A. Pitts, W. Fundamenski, S. K. Erents, Y. Andrew, A. Loarte, C. Silva and JETEFDA contributors, Nucl. Fusion 46 (2006) 82.

[5] W. Fundamenski et al. in Fusion Energy 2008 (Proc. 22nd Int. Conf. Geneva, 2008) (Vienna: IAEA) CD-ROM file EX/4-3Ra and http://www-naweb.iaea.org/napc/physics/FEC/FEC2008/html/index.htm

[6] J. H. Yu, J. A. Boedo, E. M. Hollmann, R. A. Moyer, D. L. Rudakov and P. B. Snyder, Phys. Plasmas 15 (2008) 032504.

[7] A. Kirk, S. Lisgo, E. Nardon, T. Eich, A. Herrmann, A. Kallenbach and A. Loarte, J. Nucl. Mater. 390-391 (2009) 727.

[8] D. L. Rudakov et al., J. Nucl. Mater. 390-391 (2009) 785.

[9] R. J. Maqueda, R. Maingi J.-W. Ahn, NSTX Team, J. Nucl. Mater 390-391 (2009) 843.

[10] M. Kočan et al., Plasma Phys. Control. Fusion 53 (2011) 065002.

[11] S. Carpentier, R. A. Pitts, P. C. Stangeby, J. D. Elder, A. S. Kukushkin, S. Lisgo, W. Fundamenski, D. Moulton, J. Nucl. Mater. 415 (2011) S165.

[12] R.A. Pitts et al., J. Nucl. Mater. 415 (2011) S957.

[13] R. A. Pitts et al., Plasma Phys. Control. Fusion 47 (2005) B303.

[14] B. Lipschultz et al., Nucl. Fusion 47 (2007) 1189.

[15] P. B. Snyder and H. R. Wilson, Plasma Phys. Control. Fusion 45 (2003) 1671.

[16] G. T. A. Huysmans, S. Pamela, E. van der Plas and P. Ramet, Plasma Phys. Control. Fusion 51 (2009) 124012.

[17] S. J. P. Pamela, G. T. A. Huysmans, M. N. A. Beurskens, S. Devaux, T. Eich, S. Benkadda and JET EFDA contributors, Plasma Phys. Control. Fusion 53 (2011) 054014.

[18] O. E. Garcia, N. H. Bian and W. Fundamenski, Phys. Plasmas 13 (2006) 082309.

[19] T. Eich et al., J. Nucl. Mater. 337-339 (2005) 669.

[20] W. Fundamenski et al., Nucl. Fusion 51 (2011) 083028. 
[21] T. Eich, P. Andrew, A. Herrmann, W. Fundamenski, A. Loarte, R. A. Pitts and JETEFDA contributors, Plasma Phys. Control. Fusion 49 (2007) 573.

[22] R. A. Pitts et al., J. Nucl. Mater. 390-391 (2009) 755.

[23] A. Kirk et al., Journal of Physics: Conference Series 123 (2008) 012011.

[24] A. Kirk et al., Plasma Phys. Control. Fusion 53 (2011) 035003.

[25] A. Schmid, A. Herrmann, H. W. Müller and the ASDEX Upgrade Team, Plasma Phys. Control. Fusion 50 (2008) 045007.

[26] N. Asakura et al., Plasma Phys. Control. Fusion 44 (2002) A313.

[27] B. Gonçalves et al., Plasma Phys. Control. Fusion 45 (2003) 1627.

[28] J. A. Boedo et al.,Phys. Plasmas 12 (2005) 072516.

[29] C. Silva et al., J. Nucl. Mater. 337-339 (2005) 722.

[30] A. Herrmann et al., J. Nucl. Mater. 363-365 (2007) 528.

[31] H. W. Müller et al., "Fluctuations, ELM filaments and turbulent transport in the SOL at the outer mid plane of ASDEX Upgrade”, Nucl. Fusion 51 (2011) 073023.

[32] P. Tamain, M. Kočan, J. Gunn, A. Kirk, J.-Y. Pascal and M. Price, J. Nucl. Mater. 415 (2011) S1139.

[33] W. Fundamenski, R. A. Pitts and JET EFDA Contributors, Plasma Phys. Control. Fusion 48 (2006) 109.

[34] J. P. Gunn and V. Fuchs, Quasineutral kinetic simulation of the scrape-off layer: Asymmetric ELM energy deposition on divertor plates, $35^{\text {th }}$ EPS Conference on Plasma Phys. Hersonissos, 9 - 13 June 2008 ECA Vol.32D, P-2.015 (2008).

[35] G. Manfredi et al., Parallel transport in a tokamak scrape-off layer, Proc. $38^{\text {th }}$ EPS Conf. on Plasma Physics (Strasbourg, 27 June -1 July 2011) P-4.063.

[36] R. Dux, A. Jantzer and T. Pütterich, Nucl. Fusion 51 (2011) 0530002.

[37] A. Kallenbach, Nucl. Fusion 51 (2011) 094012.

[38] W. Suttrop et al., Fusion Eng. \& Design 84 (2009) 290.

[39] W. Suttrop et al., Phys. Rev. Letters 106 (2011) 225004.

[40] A. Loarte et al., Plasma Phys. Control. Fusion 45 (2003) 1549.

[41] G. Pautasso et al., Nucl. Fusion 51 (2011) 043010. 
[42] T. Eich, A. Herrmann, J. Neuhauser and ASDEX Upgrade Team, Phys. Rev. Lett. 91 (2003) 195003.

[43] T. Eich et al., Plasma Phys. Control. Fusion 47 (2005) 815.

[44] M. Kočan et al., Rev. Sci. Instrum. 79 (2008) 073502.

[45] A. Herrmann, J. Neuhauser, V. Rohde, R. Dux, T. Eich, C. J. Fuchs, M. Y. Ye and ASDEX Upgrade team, J. Nucl. Mater. 337-339 (2005) 697.

[46] A. Loarte et al. in Fusion Energy 2006 (Proc. 21st Int. Conf. Chengdu, 2006) (Vienna: IAEA) CD-ROM file IT/P1-14 and http://www-naweb.iaea.org/napc/physics/FEC/FEC2006/html/index.htm

[47] M. Kočan et al., J. Nucl. Mater. 415 (2011) S1133.

[48] N. Ben Ayed, A. Kirk, B. Dudson, S Tallents, R. G. L. Vann, H. R. Wilson and the MAST Team, Plasma Phys. Control. Fusion 51 (2009) 035016.

[49] M. Kočan, F. P. Gennrich, A. Kendl and H. W. Müller, Ion temperature fluctuations in the ASDEX Upgrade scrape-off layer, submitted to Plasma Phys. Control. Fusion, 2011.

[50] R. A. Pitts et al., Nucl. Fusion 47 (2007) 1437.

[51] A. Kirk et al., Plasma Phys. Control. Fusion 49 (2007) 1259.

[52] A. Herrmann, ASDEX Upgrade Team, Limitations for divertor heat flux calculations of fast events in tokamaks, Europhysics Conference Abstracts (CD-ROM, Proceedings of the 28th EPS Conference on Controlled Fusion and Plasma Physics, Madeira 2001) 25A (2001) 2109.

[53] P. C. Stangeby, J. Phys. D: Appl. Phys. 18 (1985) 1547.

[54] J. Marki, R.A. Pitts, T. Eich, A. Herrmann, J. Horacek, F. Sanchez and G. Veres, J. Nucl. Mater. 363-365 (2007) 382.

[55] D. Brunner et al., J. Nucl. Mater. (2010), doi:10.1016/j.jnucmat.2010.10.026.

[56] P. C. Stangeby, The Plasma Boundary of Magnetic Fusion Devices (Bristol: Institute of Physics Publishing, 2000) p. 649.

[57] M. Kočan, Ion temperature measurements in the scrape-off layer of the Tore Supra tokamak, Ph D thesis, Nancy University, 2009. 
[58] W. Fundamenski, R. A. Pitts and JET EFDA Contributors, J. Nucl. Mater. 363-365 (2007) 319 . 